\title{
Topoisomerase VI participates in an insulator-like function that prevents H3K9me2 spreading into euchromatic islands
}

Louis-Valentin Méteignier ${ }^{1}$, Cécile Lecampion ${ }^{1}$, Florent Velay ${ }^{1}$, Cécile Vriet ${ }^{1,2}$, Laura Dimnet ${ }^{1}$, Michel Térèse $^{3}$, Martin Rougée ${ }^{4,5}$, Christian Breuer ${ }^{6}$, Ludivine Soubigou-Taconnat ${ }^{7,8}$, Keiko Sugimoto ${ }^{6}$, Fredy Barneche $e^{4,5}$ and Christophe Laloi ${ }^{1 *}$.

${ }^{1}$ Aix Marseille Univ, CEA, CNRS, BIAM, Marseille, France F-13009

${ }^{2}$ UMR CNRS 7267, University of Poitiers, 86073 Poitiers Cedex

${ }^{3}$ Independent informatician, 06100 Nice, France

${ }^{4}$ IBENS, Département de Biologie, Ecole Normale Supérieure, CNRS, PSL Research University, F75005, Paris, France

${ }^{5}$ Université Paris-Sud, Université Paris-Saclay, 91405, Orsay, France

${ }^{6}$ RIKEN Center for Sustainable Resource Science, Yokohama, 230-0045, Japan

${ }^{7}$ Institute of Plant Sciences Paris Saclay IPS2, CNRS, INRA, Université Paris-Sud, Université Evry, Université Paris-Saclay, Bâtiment 630, 91405 Orsay, France.

${ }^{8}$ Institute of Plant Sciences Paris-Saclay IPS2, Paris Diderot, Sorbonne Paris-Cité, Bâtiment 630, 91405, Orsay, France

\section{Keywords}

Euchromatin islands / Heterochromatin spreading / Insulator / Methionine Adenosyltransferase / Topoisomerase VI. 


\begin{abstract}
The organization of the genome into transcriptionally active and inactive chromatin domains requires well-delineated chromatin boundaries and insulator functions in order to maintain the identity of adjacent genomic loci with antagonistic chromatin marks and functionality. In plants that lack known chromatin insulators, the mechanisms that prevent heterochromatin spreading into euchromatin remain to be identified. Here, we show that DNA Topoisomerase VI participates in a chromatin boundary function that safeguards the expression of genes in euchromatin islands within silenced heterochromatin regions. While some transposable elements are reactivated in mutants of the Topoisomerase VI complex, genes insulated in euchromatin islands within heterochromatic regions of the Arabidopsis thaliana genome are specifically downregulated. H3K9me2 levels consistently increase at euchromatin island loci and decrease at some TE loci. We further show that Topoisomerase VI physically interacts with S-adenosylmethionine (SAM) synthase MAT3, which is required for H3K9me2 deposition. Topoisomerase VI promotes MAT3 occupancy on heterochromatic elements and its exclusion from euchromatic islands, thereby providing a mechanistic insight into the essential role of Topoisomerase VI in the delimitation of chromatin domains.
\end{abstract}




\section{Introduction}

The discovery of position effect variegation in Drosophila melanogaster paved the way towards revealing the importance of chromatin contexts in the regulation of gene expression (Wang et al, 2014; Muller, 1930). Since then, cytogenetic and molecular profiling of the epigenome, as well as topological analyses of chromatin architecture, have allowed the mechanisms involved in partitioning the gene-rich euchromatic fraction from the repeat-rich heterochromatic fraction to be elucidated. Large protein complexes specific to insulator DNA sequences contribute to partitioning chromatin domains with distinct identity at multiple scales. These complexes maintain the identity of adjacent genomic loci with antagonistic chromatin marks and functionality, and more globally influence the formation of long-range chromosomal interactions (Ali et al, 2016). Insulator binding proteins such as the CCCTC-binding factor CTCF, BEAF-32, CP190 and Mod have been best described in Drosophila where they play critical roles in the definition of chromatin and transcriptional status. In vertebrates, CTCF is the only known insulator binding protein homologue. CTCF is enriched at insulator DNA sequences that define large topological domains of the genome (Bickmore \& van Steensel, 2013; Dekker \& Misteli, 2015; Dixon et al, 2012) and, in some cases, define boundaries between adjacent chromatin domains with distinct features (Bonev \& Cavalli, 2016). Surprisingly, CTCF orthologs cannot be identified in many eukaryotic organisms, including plants (Heger et al, 2012). Moreover, very few studies support the presence of insulator DNA sequences or insulator-like regions in plants (Wang et al, 2015a; Singer et al, 2011; Liu et al, 2017), and insulator binding factors remain to be identified. This contrasts with the observation that Arabidopsis thaliana and other plant species display highly indexed chromatin states along the genome, with well-defined chromatin signatures around transcriptionally active or repressed genes, as well as close relationships between chromatin composition and genome topology in the nuclear space (Liu \& Weigel, 2015; SequeiraMendes \& Gutierrez, 2016). In Arabidopsis, heterochromatin is found on hundreds of transposable elements (TEs) mostly confined within the pericentromeric regions and at a few knob structures that tend to associate through long-distance interactions in the nuclear space (Grob et al, 2013, 2014; Feng et al, 2014; Veluchamy et al, 2016; Liu et al, 2016). As a result, in Arabidopsis interphase nuclei most cytologically visible heterochromatin is condensed within 8 to 10 conspicuous foci that are referred to as chromocenters (Fransz \& De Jong, 2011; Simon et al, 2015; Del Prete et al, 2014). Consistent with their heterochromatic nature, chromocenters are refractory to transcription and contain highly methylated DNA (Fransz et al, 2002) as well as histone modifications such as H3K9me2 and H3K27me2 (Soppe et al, 2002; Mathieu et al, 2005). Nonetheless, many expressed genes exhibiting euchromatic features appear to be located in close vicinity to large heterochromatic regions in the Arabidopsis genome, notably within the pericentromeric and knob regions (Lippman et 
al, 2004; Vergara \& Gutierrez, 2017). The mechanisms by which gene-containing euchromatic islands (Els) are insulated from neighboring heterochromatin regions and how their transcriptional capacities are preserved in such chromatin contexts are largely unknown. In this study, we have unveiled an essential function of the plant Topoisomerase VI complex in preserving the functional and structural identity of Els.

DNA topoisomerases are enzymes that introduce transient DNA breaks to resolve topological constraints that arise during multiple cellular processes such as replication, transcription, recombination and chromatin remodeling. The plant Topo $\mathrm{VI}$, a type II topoisomerase first identified in the archaeon Sulfolobus shibatae (Bergerat et al, 1994, 1997), was initially implicated in various biological processes involving endoreduplication, such as root hair growth (Schneider et al, 1998, 1997; Sugimoto-Shirasu et al, 2005), hypocotyl elongation (Sugimoto-Shirasu et al, 2005) and nodule differentiation (Yoon et al, 2014). Topo $\mathrm{VI}$ forms an $\mathrm{A}_{2} \mathrm{~B}_{2}$ heterotetramer whose $\mathrm{A}$ and $\mathrm{B}$ subunits are encoded by single genes in Arabidopsis, AtTOP6A/CAA39/AtSPO11-3/RHL2/BIN5/AT5G02820 and AtTOP6B/RHL3/BIN3/HYP6/HLQ/AT3G20780, respectively (Hartung \& Puchta, 2001; Hartung et al, 2002; Yin et al, 2002; Mittal et al, 2014; Sugimoto-Shirasu et al, 2002). Two additional subunits named ROOT HAIRLESS 1 (RHL1/HYP7/AT1G48380) and BRASSINOSTEROID-INSENSITIVE 4 (BIN4/MID/AT5G24630) (Breuer et al, 2007; Kirik et al, 2007; Sugimoto-Shirasu et al, 2005) are essential for the Arabidopsis Topo VI function and appear to be evolutionarily conserved in plants and in other eukaryote groups, whilst their precise functions remain unclear. However, BIN4 shares sequence similarity with the C-terminal region of animal Topo $I \alpha$, which seems to have regulatory functions (Meczes et al, 2008; Onoda et al, 2014; Gilroy \& Austin, 2011), and exhibits stable DNA binding in vitro (Breuer et al, 2007). Therefore, it has been proposed that BIN4 may have a regulatory role in the plant Topo VI complex, presumably by holding the substrate DNA through its AT-hook motif (Breuer et al, 2007; Kirik et al, 2007).

In recent years, evidence has accumulated that topoisomerases have more diverse and specialized functions than previously thought (Pommier et al, 2016). In particular, transcriptomic analyses of several Topo VI mutants revealed that Topo VI influences the expression of many nuclear genes, including genes regulated by phytohormones (Yin et al, 2002; Mittal et al, 2014) or by reactive oxygen species (Jain et al, 2006, 2008; Simkova et al, 2012). A function of Arabidopsis Topo VI as a chromatin-remodeling complex has also been speculated (Yin et al, 2002). This hypothesis has since been supported by the observation that loss of the Topo VI B subunit in hlq mutant plants leads to the mis-expression of numerous adjacent genes, hence possibly triggering positional or chromatin context dependent transcriptional defects (Mittal et al, 2014). This is further supported by the 
implication of the BIN4 subunit in heterochromatin organization, as observed by smaller and diffuse chromocenters in interphase nuclei of plants bearing the severe mid mutation (Kirik et al, 2007).

Here, we reveal that Arabidopsis Topo VI is required for chromocenter formation and for efficient silencing of some heterochromatic TEs, but has an antagonistic effect on genes localized in euchromatic islands (Els). Downregulation of El genes in Topo VI mutant plants is associated with an enrichment of the H3K9me2 heterochromatic mark. We further report that the BIN4 subunit of Topo VI directly interacts with S-adenosylmethionine (SAM) synthetase 3 / Methionine Adenosyl transferase 3 (MAT3). Similarly to Topo VI knockdown plants, mat3 knockdown mutants exhibit derepression of heterochromatic TEs and a decrease in H3K9me2. Furthermore, we show that MAT tethering to heterochromatin diminishes in a hypomorphic Topo VI mutant, leading to increased MATs localisation to Els. This provides a mechanistic insight into the simultaneous local decrease of H3K9me2 in heterochromatin and increase of H3K9me2 at Els in the Topo VI mutant. We therefore propose that Topo VI has a prominent role in the delimitation of chromatin boundaries, localizes SAM synthesis onto specific regions of the genome, and collectively has an essential role in the establishment of distinct chromatin domains.

\section{Results}

\section{Topoisomerase $\mathrm{VI}$ is required for heterochromatin organization}

Kirik et al. reported that interphase nuclei of the severe mid mutant in the BIN4/MID subunit presents smaller and less defined chromocenters (CCs) compared to the wild-type (wt), suggesting that heterochromatin organization is affected by the mid mutation (Kirik et al, 2007). However, this phenotype was not reported in the allelic bin4-1 mutant, which also has a severe phenotype (Breuer et al, 2007). Therefore, to unequivocally confirm the role of the Arabidopsis Topo VI complex in nuclear organization, we analyzed the nuclear phenotypes of hypomorphic and amorphic mutants of the AtTOP6A subunit, caa39 and rh/2-1, and of the BIN4/MID subunit, a BIN4 knockdown line (BIN4 KD, see below) and bin4-1, by DAPI DNA staining and immunolocalization of heterochromatin hallmarks. Both the caa39 and rh/2-1 mutants exhibited strong alterations in heterochromatin organization with largely decondensed chromocenters (Fig 1A, top panel, and Fig S1A). Likewise, nuclei of epidermal and mesophyll cotyledon cells from BIN4 KD and bin4-1 did not harbor conspicuous chromocenters (Fig S1A), as previously reported for the mid allelic mutants (Kirik et al, 2007). In contrast, the nuclear phenotype of shoot apical meristematic cells is similar in wt, caa39, rh/2-1, bin4-1 and BIN4 KD lines, with equal proportions of nuclei with conspicuous (type 1) or diffuse (type 2) chromocenter profiles (Fig S1A, meristem panel, and S1B). Consistent with its role in 
endocycles but not in mitosis (Breuer et al, 2007; Kirik et al, 2007; Sugimoto-Shirasu et al, 2002, 2005), these defects indicate that Topo VI is required for chromatin organization of differentiated cells, but less of actively dividing cells. Immunofluorescence analysis of the heterochromatin hallmark H3K9me2 confirmed the large extent of heterochromatin decondensation in caa39 Topo VI mutant plants (Fig 1A). Immunoblot analyses further showed that the global level of H3K9me2 is not affected in caa39 seedlings (Fig 1B). Likewise, 5-methylcytosine (5-meC) immunolabeling also revealed a dispersed signal in caa39 nuclei (Fig 1C) whereas an anti-5-meC ELISA showed overall similar levels of 5-meC in wt and caa39 as compared to ddm1-8 seedlings (Fig 1D). These results suggest that the marked alteration of chromocenter morphology does not result from a global decrease in heterochromatin hallmarks in caa39.

A

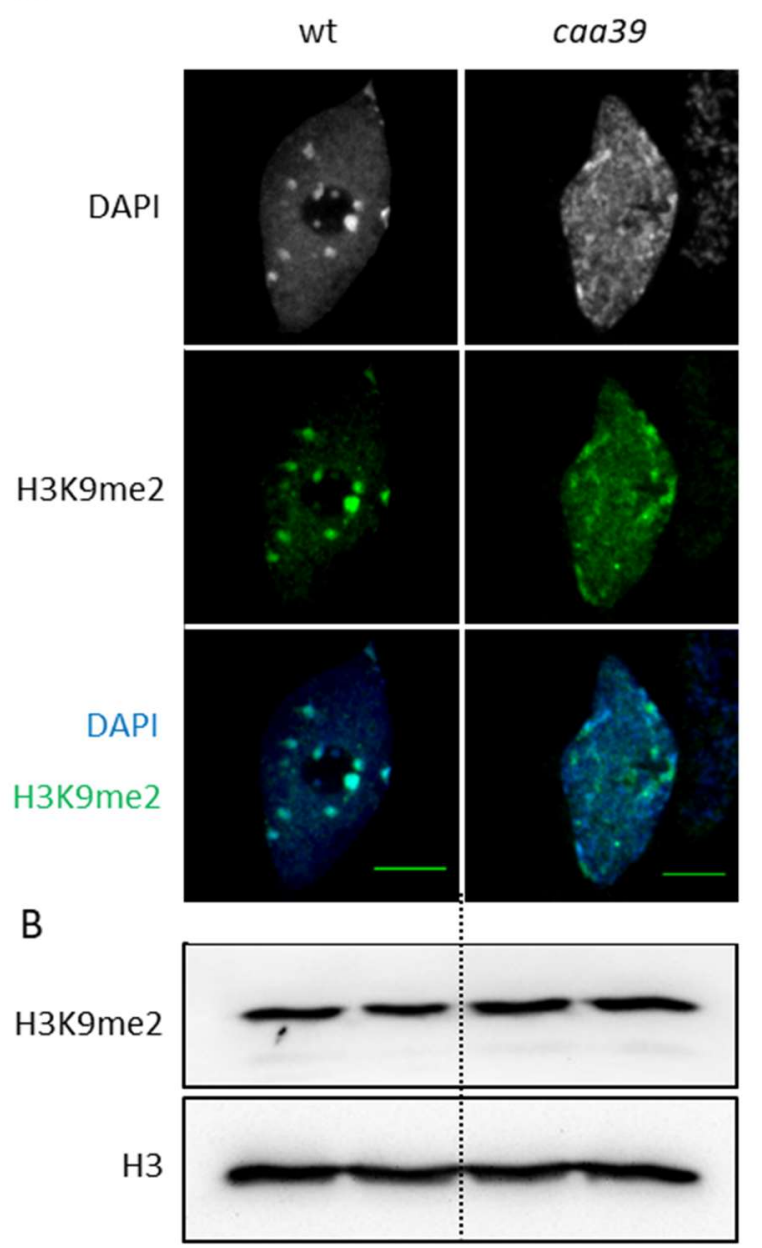

C

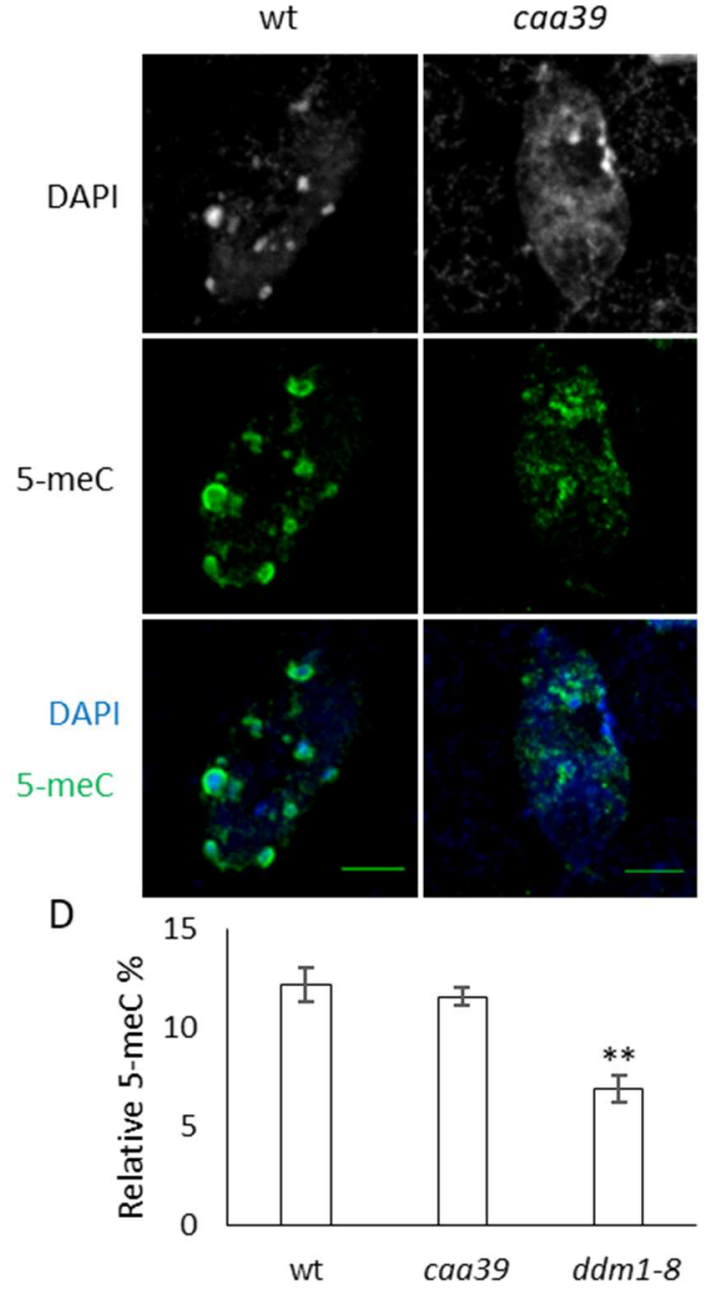

Figure 1. Topoisomerase $\mathrm{VI}$ is required for heterochromatin organization. (A) Representative nucleus $(n>30)$ from 6 day-old wt and caa39 cotyledon epidermal cells stained with DAPI and showing indirect immunolocalization of H3K9me2. Scale bar: $5 \mu \mathrm{m}$. (B) Two independently prepared nuclear extracts of wt and caa39 were immunoblotted against $\mathrm{H} 3$ or H3K9me2, as indicated. (C) 
Same as (A) for 5-meC localization. (D) Elisa assay to quantify total 5-meC in 6 day-old cotyledons of wt, caa39 and ddm1-8. ${ }^{* *}: P<0.005$ (Student's $t$-test).

\section{Topo VI is required for the silencing of heterochromatic transposable elements}

A role for Arabidopsis Topo VI in heterochromatin-dependent transcriptional gene silencing was highlighted by the reactivation of TRANSCRIPTIONALLY SILENT INFORMATION (TSI) in mid mutant plants (Kirik et al, 2007). However, reactivation was not observed in the bin4-1 allelic mutant (Breuer et al, 2007). Therefore, to unambiguously assess the involvement of Topo $\mathrm{VI}$ in transcriptional silencing and get a more global understanding of Topo VI influence on TE repression, we performed a RNA-seq analysis of caa39 and wt transcripts. Multiple heterochromatic TEs (176 TEs with log2(FC) > 2), particularly from the LTR/Gypsy, LTR/Copia and En-Spm/CACTA superfamilies (Underwood et al, 2017), are reactivated in caa39 plants (Fig 2A, Appendix Table S1). Conversely, 91 TEs are repressed in caa39 $(\log 2(\mathrm{FC})<-2)$ and unlike reactivated TEs, these repressed TEs are rarely in the most inaccessible and repressive heterochromatin state 9 (Fig S2A). To test for TE silencing defects in other Topo VI mutants, we selected several de-repressed heterochromatic TEs loci (Appendix Table S1) for which robust primer design was feasible, and measured their relative transcript abundance by RTqPCR in rh/2-1 and bin4-1 mutants along with the caa39 and wt lines. A clear increase in TE transcript abundance was observed for all three tested Topo VI mutant lines (Fig 2B).

Although we found no global decrease of H3K9me2 and 5-meC in caa39 (Fig 1B and 1D), more subtle local changes could account for TE reactivation. We first assessed DNA methylation levels at individual TEs in Topo VI mutants as compared to wt and ddm1-8 plants by digestion with the methylation-dependent restriction enzyme McrBC. As expected, very efficient digestion of TEs was observed in wt but not in ddm1-8 plants, reflecting a nearly complete loss of DNA methylation over multiple TEs in this hypomethylated mutant line (Fig 2C). In sharp contrast, we observed wt levels of DNA methylation for all tested loci in caa39 and bin4-1 plants. This trend was confirmed in different sequence contexts ( $\mathrm{CG}, \mathrm{CHG}$ and $\mathrm{CHH}$ ) by using the methylation-sensitive restriction enzymes Hpall, Mspl and HaellI (Fig S3). Similar DNA methylation levels of TEs in wt and caa39 were then confirmed genome-wide by whole-genome bisulfite sequencing, in all three contexts (Fig 2D). We concluded that TE de-repression in Topo VI mutants could not be accounted for by a global decrease of DNA methylation in cis. Next, we measured the level of H3K9me2 at TEs, which could be performed only with the caa39 hypomorphic mutant, owing to the extreme dwarf phenotype of the rh/2-1 and bin4-1 null mutants. ChIP-qPCR analyses revealed a modest decrease in H3K9me2 at some but not all tested TEs in caa39 as compared to wt plants (Fig 2E). ChIP-seq analysis of H3K9me2 levels 
in wt and caa39, normalized to H3 levels in each line, confirmed the slight decrease of H3K9me2 at AT3TE60425 and AT4TE15030, but not at AT2TE15415 and AT4TE16900 (Fig S2B-C), and revealed a consistent decrease or increase of H3K9me2 on upregulated or downregulated TEs, respectively (Fig 2F). Collectively, the results presented in figure 1 and 2 suggest that TE reactivation results from a combination of moderate and local decreases of $\mathrm{H} 3 \mathrm{~K} 9 \mathrm{me} 2$ and heterochromatin reorganization. Moreover, the expression pattern of TEs correlates well with corresponding changes in their H3K9me2 content but not with DNA methylation changes.
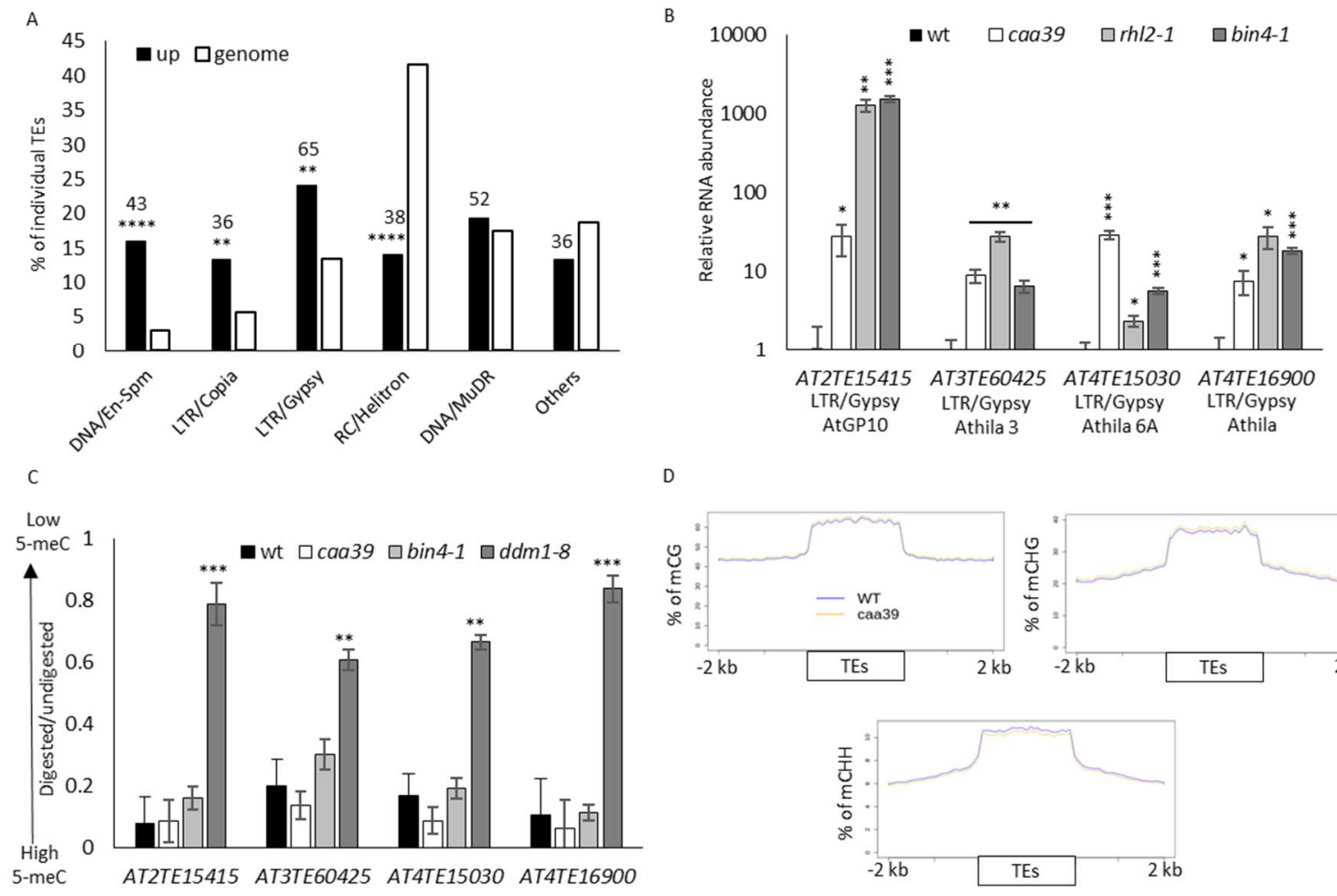

D
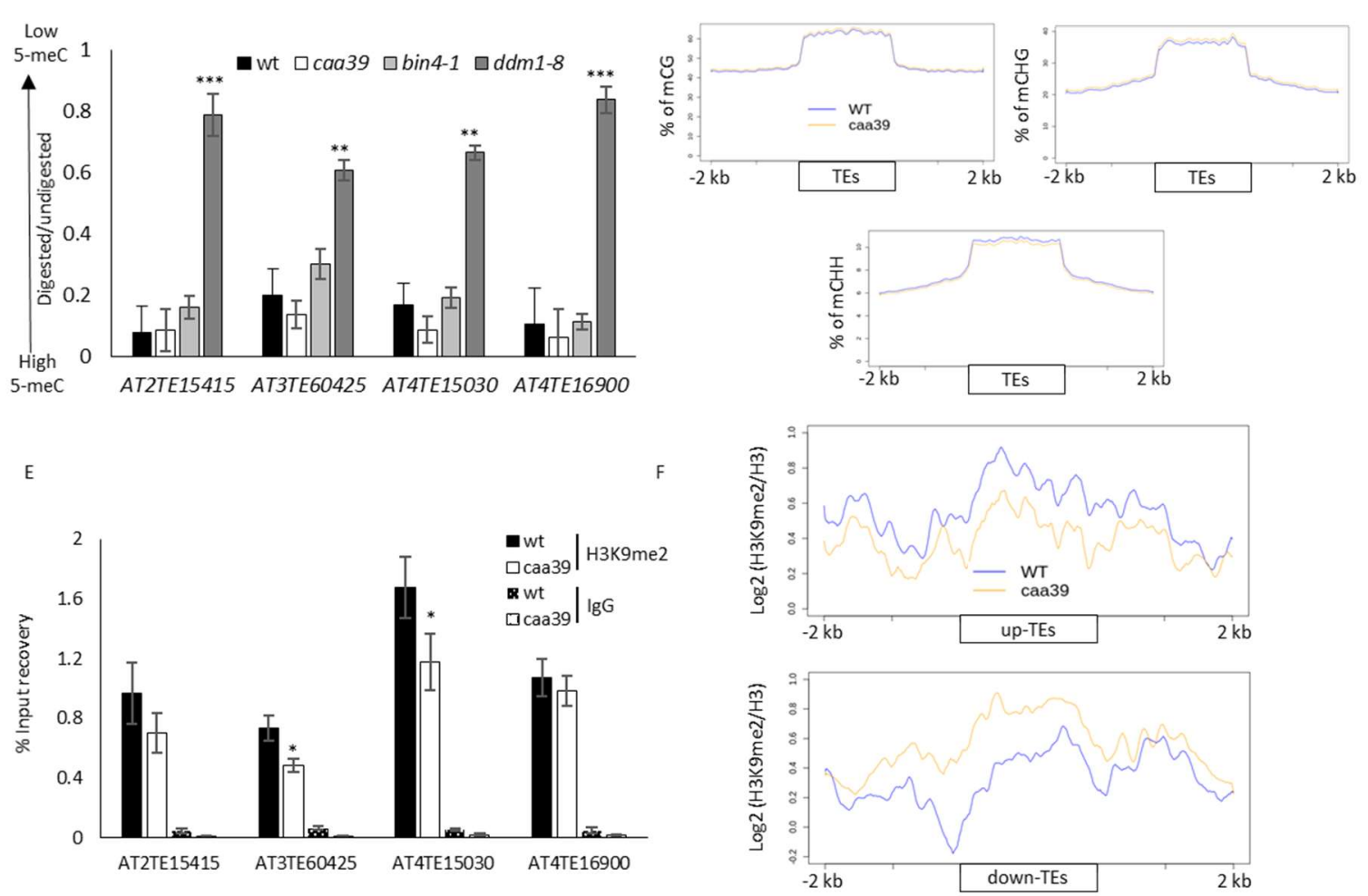

Figure 2. Topo $\mathrm{VI}$ is required for the silencing of transposable elements. (A) Bar chart showing the proportions of reactivated TE superfamilies in caa39 compared to the general proportion of TEs in the genome. The relative percentage is shown for each superfamily and the absolute number of reactivated TEs is noted above each bar. ${ }^{* *}$ : $\mathrm{P}<0.005 ;{ }^{* * * *}: \mathrm{P}<0.0001$ (Chi-squared test). (B) RT- 
qPCR confirmation of the reactivation of selected TEs in caa39, rh/2-1 and bin4-1. Error bars: \pm SEM of three biological replicates. *: $P<0.05$; ${ }^{* *}: P<0.005 ;{ }^{* *}: P<0.0005$ (Student's $t$-test). (C) DNA from indicated genotypes were extracted and digested with McrBC. The mean ratio of digested over undigested DNA from three biological replicates is shown. Error bars: \pm SEM of three biological replicates. *: $P<0.05 ;{ }^{* *}: P<0.005 ;{ }^{* *}: P<0.0005$ (Student's $t$-test). (D) Average distribution of methylated cytosine in $\mathrm{CG}, \mathrm{CHG}$ and $\mathrm{CHH}$ contexts over up- or downregulated TEs. Two independent replicates for each genotype were performed. (E) ChIP-qPCR of H3K9me2 at selected TEs. Error bars: \pm SEM of three biological replicates. ${ }^{*}: P<0.05 ;{ }^{* *}: P<0.005 ;{ }^{* * *}: P<0.0005$ (Student's $t$-test). (F) Average distribution of $\mathrm{H} 3$-normalized $\mathrm{H} 3 \mathrm{~K} 9 \mathrm{me} 2$ along upregulated or downregulated TEs and $2 \mathrm{~kb}$ flanking regions. Two (H3) and three (H3K9me2) independent biological replicates for each genotype were performed.

\section{Unlike TEs, genes interspersed within pericentromeric and chromosome 4 knob large heterochromatin regions are massively downregulated in Topo VI mutants}

We then used the online positional gene enrichment tool (De Preter et al, 2008) to investigate the genomic distribution of misregulated genes identified in our RNA-seq analysis of caa39 (Appendix Table S2). This analysis revealed that the 500 most downregulated genes are strikingly over-represented in pericentromeric regions (PRs) and in the heterochromatic knob of chromosome 4 ( $h k 4 S$, Fig 3A). In these regions, 94\% (181/193) of the non-TE genes that are differentially expressed in caa39 are downregulated (Appendix Table S2). In contrast, the 500 most highly upregulated genes displayed no preferential localization (Fig S4A). To determine whether this effect is robust in other Topo VI mutant lines, we first examined the expression profiles of bin4-1 and a second allelic mutant, bin4-2, from microarray data that were generated during the initial characterization of these two allelic lines (Breuer et al, 2007). Despite the fact that bin4-1 and bin4-2 are knock-out mutants that have much more severe developmental defects than caa39, and although two different technical platforms have been used (RNA-seq for caa39 vs Affymetrix ATH1 microarrays for bin4-1 and bin4-2), we found a good correlation between the different transcriptomes (Fig S4B). In particular, 91\% (72/79) of the PR genes that are repressed in caa39 and are detected in both RNA-seq and microarray experiments are also down-regulated in bin4-1 or bin42 (Fig 3B, Appendix Table S2). We examined further the expression of seven pericentromeric genes distributed over the five chromosomes and strongly repressed in caa39, by RT-qPCR in caa39, bin4-1 as well as in rh/2-1 plants. These genes were found to be downregulated in all mutants, except for AT4G06634 and AT4G07390 that were not significantly repressed in rh/2-1 and bin4-1 (Fig 3C). This could possibly be due to secondary effects of the bin4-1 and rh/2-1 amorphic mutations as compared to the less severe caa39 mutation. In order to test this hypothesis, we took advantage of the availability of an Arabidopsis BIN4 co-suppressed transgenic line identified during the process of 
generating lines overexpressing BIN4-CFP. Rather than exhibiting BIN4 upregulation, this BIN4 KD homozygous, monoinsertional transgenic line, shows downregulation of BIN4 (Fig S5A-B) and develops a phenotype similar to caa39 (Fig S5C). In this line, all tested pericentromeric genes were at least as much downregulated as in caa39, with a more pronounced effect than in the bin4-1 and rh/21 knockout mutants (Fig 3C). These observations indicate that Topoisomerase $\mathrm{VI}$ is required to maintain transcriptional control of both genes and TEs in pericentromeric and $h k 4 S$ regions, possibly acting as a chromatin architectural factor.
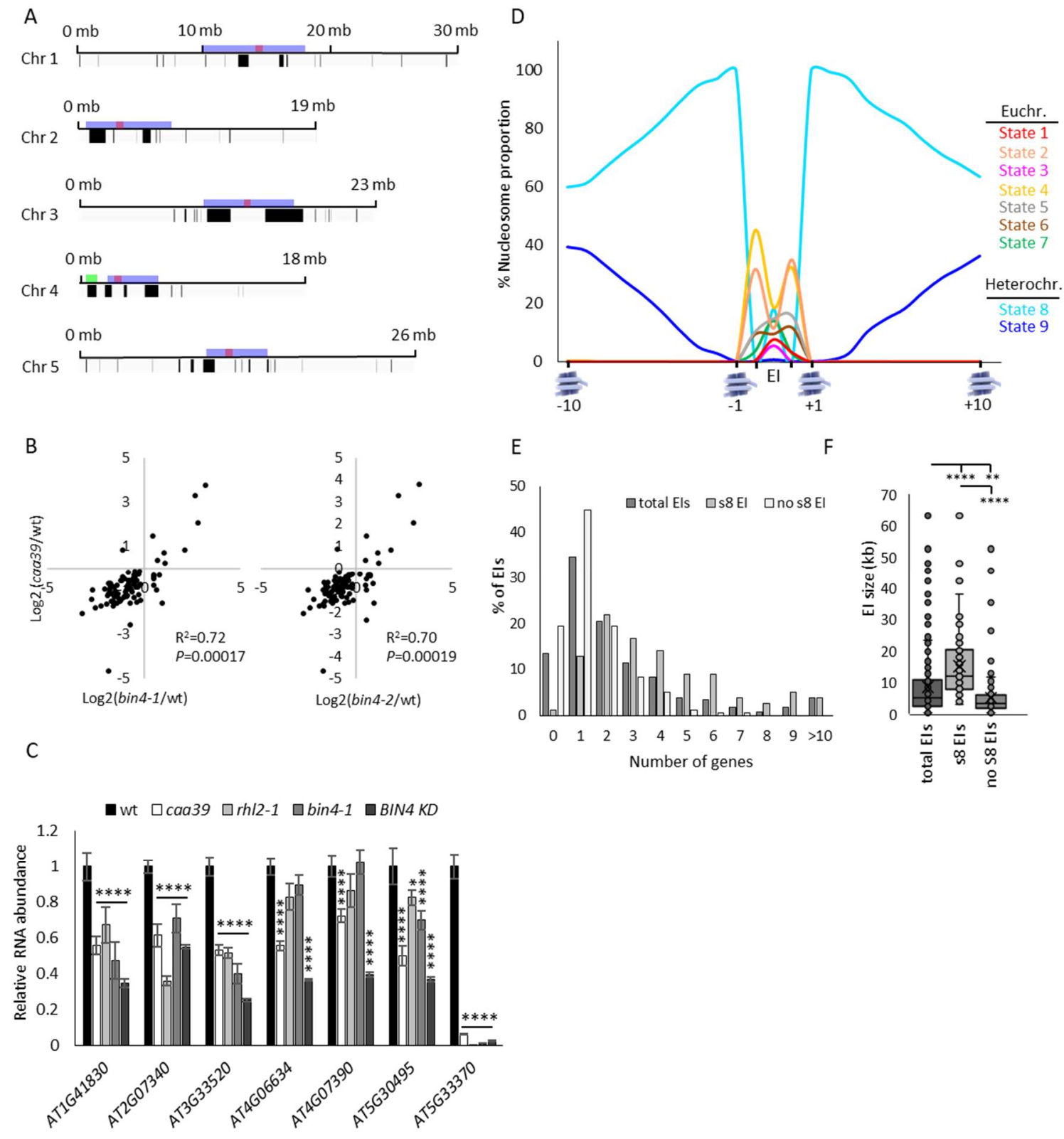

Figure 3. Genes in euchromatic islands within heterochromatic pericentromeric and chromosome 4 knob regions are repressed in Topo VI mutants. (A) Positional Gene Enrichment analysis of the top 
500 most downregulated genes in caa39. The bed files corresponding to coordinates of the widest enriched regions (FDR $<0.05)$ were visualized with a genome browser. Black lines correspond to enriched regions, blue boxes correspond to pericentromeric regions as defined by Yelina et al. (2012), green box to the knob and red to centromeres. (B) Scatter plots and pearson correlations of differentially expressed $(P<0.05)$ pericentromeric genes in caa39 and bin4-1 or bin4-2. (C) RT-qPCR of selected pericentromeric genes in caa39, rh/2-1, bin4-1 and BIN4 KD at 6 days post-germination. Error bars: \pm SEM of three biological replicates. ${ }^{* *}: P<0.005 ;{ }^{* *}: P<0.0005 ; * * * *: P<0.0001$ (twoway ANOVA, Dunnett's test). (D) The proportion of each chromatin state was computed for ten nucleosomes on each side of the islands, along with the first nucleosome within each island. The proportions of euchromatic states within the islands correspond to the averaged proportions of all island nucleotides. El: Euchromatic islands. (E) El gene content in total, state 8- or no state 8containing Els. (F) Els size distribution for total, state 8- or no state 8-containing Els. ${ }^{* *}: P<0.005$; $* * * *: P<0.0001$ (ANOVA, Tukey's test).

\section{Downregulated genes within heterochromatic regions are localized in small euchromatic islands}

We then asked whether the inverse expression patterns of genes and TEs in pericentromeric and $h k 4 S$ regions in Topo VI mutants could be ascribed to their different chromatin landscapes. We first inspected the individual chromatin landscape of the seven downregulated pericentromeric genes confirmed by RT-qPCR (Fig 3C), using the nine chromatin states defined by Sequeira-Mendes et al. (Sequeira-Mendes et al, 2014). Euchromatin states 2, 1 and 3 characterize the proximal promoter, the transcriptional start site, and the start of coding sequence, respectively. The intragenic states 6 and 7 are characteristic of the transcriptional termination site and gene body of long transcribed genes, respectively. States 4 and 5 are highly enriched in H3K27me3 (a Polycomb-Repressive Complex 2 (PRC2)-based repressive histone modification) and are usually found in intergenic regions and PRC2-targeted genes. Lastly, the two types of heterochromatin states, 8 and 9, are enriched in H3K9me2, but in contrast with state 8 , state 9 preferentially defines pericentromeric heterochromatin and is devoid of H3K27me3 (Sequeira-Mendes et al, 2014). Strikingly, all inspected loci share common features: a typical euchromatin context whose proximal environment is composed of heterochromatic state 8 and whose distal environment is of heterochromatic state 9 (Fig S6A). Overall, these observations suggested that caa39 downregulated genes might be part of bona fide euchromatic islands (Els).

To generate a comprehensive view of their structural features in the genome, we systematically investigated the pericentromeric and $h k 4 S$ heterochromatic regions defined in Figure S6B. We designed a script to extract all Els surrounded by chromatin states 8 and 9 and identified 232 Els containing $540 \mathrm{El}$ genes this way, among which 6 correspond to unsequenced gaps (https://apps.araport.org/jbrowse) and were discarded in subsequent analyses (Appendix Table S3). 
Looking for Els directly flanked by state 9 chromatin did not increase the number of Els identified, showing that chromatin state 8 is always present in the proximal border (Fig 3D, Fig S6C). In contrast, the number of detected Els started to decrease to 215 when considering at least 2 consecutive state 8 nucleosomes, indicating that 11 Els have only one proximal nucleosome in state 8 (Fig S6C). With respect to state 9 , the number of extracted islands began to drop from 4 consecutive nucleosomes (Fig S6C). To gain a better insight into the chromatin landscape of Els, we analyzed the proportion of chromatin states covering Els and ten nucleosomes on each side of Els. This analysis revealed that Els are mainly composed of chromatin states 1 to 7 with state 8 in 78 Els (Fig 3D, Appendix Table S3). A majority of Els are short (S-Els) and contain 1 or 2 genes (Fig. 3E), and the 78 state 8-containing Els were in average significantly longer (Fig 3F; Appendix Table S4) and contained more genes (Fig 3E, Appendix Table S3) than state 8-free Els.

\section{Topo VI prevents the spreading of H3K9me2 into euchromatic islands}

Given the general repression of El genes and the local decrease of H3K9me2 at some TE loci without affecting the global level of H3K9me2 (Fig 1B), we hypothesized that El gene repression might result from ectopic spreading of this silencing mark over Els. This was first tested on several EI genes by ChIP-qPCR analysis of H3K9me2 levels in wt and caa39. H3K9me2 levels were very low, barely above background, in wt, consistent with the fairly high level of expression of these genes (Fig 4A). In contrast, a clear increase of H3K9me2 was observed in caa39 (Fig 4A). Therefore, we further tested the spreading of H3K9me2 over Els on a genome-wide scale by ChIP-seq analysis of H3K9me2 levels in wt and caa39, normalized to H3 levels in each line. Analysis of the wt profile of S-Els (SEls<6kb) showed a chromatin landscape where H3K9me2 was barely detected, flanked by regions with high H3K9me2 levels (Fig 4B). Consistent with the minor decrease observed only on some TEs presented in Figure 2, the H3K9me2 level is globally not lower in El-flanking sequences in caa39 as compared to wt. In contrast, a clear increase was observed within S-Els, which was highly significant all along S-Els (Mann-Whitney test, $P<0.01$ ), suggesting that Topo VI prevents H3K9me2 spreading across natural boundaries (Fig 4B). H3K9me2 spreading into large Els (L-Els) was also highly significant and particularly pronounced on L-Els boundaries (Fig. 4C). Inspection of meta-profiles for each chromosome revealed highly significant H3K9me2 spreading into Els (Fig S7A), that was observed for all replicates (Fig S7B). We then compared Els to randomly generated euchromatin regions of the same length and observed that the most significant increase of H3K9me2 is located in Els, although other chromosome arm regions may also gain slight amounts of H3K9me2 (Fig S8A and 
B). This shows that Topo VI exerts its H3K9me2 spreading inhibitory function mainly within heterochromatin regions.

To further strengthen our analysis, we applied diffReps on each replicate individually (Shen et al, 2013) to confirm differential enrichment of H3K9me2 in Els of caa39 and wt (Appendix Table S5). This very stringent and not very sensitive analysis (essentially due to the fact that H3K9me2 peaks were barely detectable in wt and that H3K9me2 spreading does not appear to be sequence-specific) could still reveal increased levels of H3K9me2 in some Els of caa39, but not all (90.4\% in replicate 2, $70.5 \%$ in replicate $3,21.7 \%$ in replicate 1; Appendix Table S3). The limited number of Els identified this way in replicate 1 can be attributed to the fact that it was less deeply sequenced than replicates 2 and 3 (GSE103924). Despite that, there was a large overlap between replicates (Fig S7C). We then extracted the number of significantly (g test, $P<0.01$ ) up or down H3K9me2 peaks in caa39 versus wt, in each individual replicate (Appendix Table S5, sheet 6). A similar percentage of significantly up or down H3K9me2 peaks was observed at the genome scale by this method (Fig 4D), in agreement with the fact that the global level of H3K9me2 is not overtly affected in caa39. In contrast, the proportion of peaks was significantly shifted toward gains of H3K9me2 within Els (Fig 4D), here again confirming the specific role of Topo VI in safeguarding El genes from ectopic H3K9me2 marking.

Globally, these data show that Topo VI is required to prevent elevated H3K9me2 levels within Els, presumably by preserving sharp boundaries between these insulated elements to avoid pervasive spreading of heterochromatin from flanking regions. We further confirmed such a barrierlike function on a S-El containing a single gene, At1g41830, by ChIP-qPCR analysis of H3K9me2. Scanning of six different loci along this region (Fig $4 \mathrm{E}$ ) in independent experiments confirmed the increased H3K9me2 levels within the island body, but also a decrease in one neighboring heterochromatic border (Fig 4E), similarly to what was observed in the ChIP-seq experiment replicates for this El (Fig $4 \mathrm{I}$ and Fig S8C) and other inspected Els (Fig S8C). 

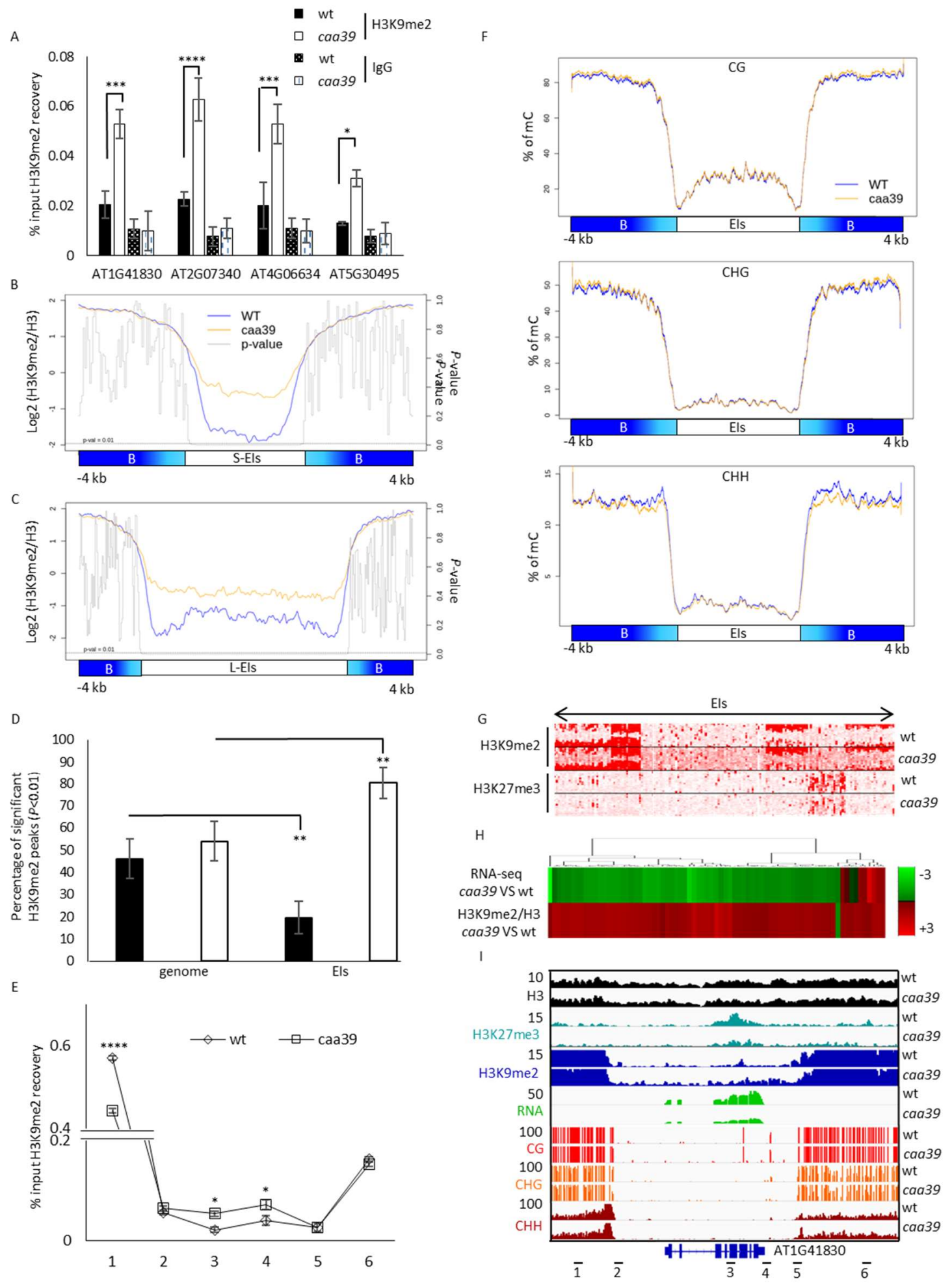

Figure 4. Topo VI prevents the invasion of euchromatic islands by H3K9me2. (A) ChIP-qPCR analysis of H3K9me2 at El genes. Error bars: \pm SEM of two biological replicates. ${ }^{*}: P<0.05 ;{ }^{* * *}: P<0.0005$; $* * * *: P<0.0001$ (two-way ANOVA, Fisher's test). (B) Average distribution of H3-normalized H3K9me2 along short euchromatic gene islands and $4 \mathrm{~kb}$ flanking regions. Two (H3) and three (H3K9me2) independent biological replicates for each genotype were performed. $P$-value was computed for each aggregated point by using a Mann-Whitney test. (C) Same as (B) long Els. (D) Bar chart showing the average percentage of down or up H3K9me2 peaks in the genome or in Els. Error 
bars: \pm SEM of three biological replicates. ${ }^{*}: P<0.05 ;{ }^{* *}: P<0.005 ;{ }^{* *}: P<0.0005$ (two-way ANOVA, Fisher's test). (E) ChIP-qPCR validation of a single-gene island for H3K9me2. Error bars: \pm SEM of three biological replicates. *: $P<0.05$; ${ }^{* * *}: P<0.0001$ (two-way ANOVA, Fisher's test). (F) Average distribution of methylated cytosine in $\mathrm{CG}, \mathrm{CHG}$ and $\mathrm{CHH}$ contexts over Els. Two independent replicates for each genotype were performed. (G) K-means linear clustering of H3K9me2 and H3K27me3 tag densities across Els and their 900 nt flanking borders as revealed by seqMINER in caa39 and wt. $(\mathrm{H})$ Heatmap correlation clustering of El genes with significant changes in RNA-seq and H3K9me2/H3 ChIP-seq. (I) Integrative Genomics Viewer screenshot of H3, H3K9me2, H3K27me3, RNA, CG, CHG and CHH methylation profiles in wt and caa39 on a single gene-containing island. Each track is normalized against corresponding input samples (ChIP-seq) and by the sequencing depth. Numbers indicate the position of primers used in (E).

\section{El gene repression is linked to $\mathrm{H} 3 \mathrm{~K} 9 \mathrm{me} 2$ spreading}

We then tried to evaluate the relative contribution of $\mathrm{H} 3 \mathrm{~K} 9 \mathrm{me} 2$ increase to the downregulation of El genes, compared to other heterochromatic and repressive marks. Firstly, because H3K9me2 and non-CG (CHG and CHH) DNA methylation are strongly inter-dependent (Stroud et al, 2014), we tested whether H3K9me2 spreading into Els might in turn, or reciprocally, affect DNA methylation. Whole-genome bisulfite sequencing revealed that DNA methylation levels in all sequence contexts were unaltered in caa39 relative to wt in Els (Fig 4F and I, Fig S8C). Differentially methylated region (DMR) analyses confirmed that there was no global significant increase of DNA methylation in Els (Dataset S1). Surprisingly, despite CHGs being known to be methylated through a feedback loop with H3K9me2, DMR analyses rather revealed a decrease of CHG, with 51 hypo-CHG DMRs and 16 hyper-CHG DMRs observed in Els of caa39 (Dataset S1). Therefore, DNA methylation does not seem to contribute to the down-regulation of gene expression in the Els and.

Secondly, because El boundaries are enriched in H3K27me3-marked heterochromatin state 8 , and that H3K27me3 is also found on the proximal promoter (chromatin state 2) and transcribed region of many euchromatic genes (chromatin state 5, silenced genes) (Sequeira-Mendes et al, 2014), we also performed a genome-wide H3K27me3 analysis by ChIP-seq. Interestingly, we observed a globally inverted tendency as compared to H3K9me2 profiles. Indeed, average H3K27me3 levels were locally and significantly decreased within Els (Fig S9A-B). We further documented such a local decrease of H3K27me3 on the small El containing At1g41830, by ChIP-qPCR (Fig S9C). Consistent with this, K-means linear clustering revealed that H3K27me3 decrease could not be generalized to all Els, as it marks only a small subset of Els in wt (Fig 4G). However, more global and significant H3K27me3 increased levels were observed in neighboring caa39 heterochromatin (Fig S9A-B). Thus, on one hand, H3K27me3 does not seem to contribute to the global down-regulation of El genes, and its local decrease on some El genes might even counterbalance the effect of H3K9me2 
increase in a few Els. On the other hand, we observed more global and significant increases of H3K27me3 in heterochromatin regions at the border of Els, suggesting that Topo $\mathrm{VI}$, in addition to its role in preventing ectopic H3K9me2 marking within Els, also prevents H3K27me3 to spread into heterochromatin borders of Els.

Finally, we directly compared El gene expression with H3K9me2 changes. A vast majority $(90 \%)$ of El genes that show significant changes $(P<0.05)$ in either RNA-seq or ChIP-seq analyses are repressed and possess enhanced levels of H3K9me2 (Fig $4 \mathrm{H}$ and I, Fig S10). Interestingly, the most repressed genes are short and tend to also possess the clearest increases of $\mathrm{H} 3 \mathrm{~K} 9 \mathrm{me} 2$ (Fig $4 \mathrm{H}$, Fig S10). Taken together, these results suggest that H3K9me2 increase plays a major role in the reduced expression of El genes, although higher order chromatin structure, changes in other histone modification, and other indirect effects of the caa39 mutation may contribute to gene expression changes.

\section{The Topo VI subunit BIN4 physically associates with MAT3}

To gain knowledge on the molecular mechanism by which Topo VI contributes to the delimitation of chromatin boundaries, we used the BIN4 subunit as a bait to screen a yeast two hybrid (Y2H) cDNA library (Hybrigenics). A strong interaction with the Topo VI subunit RHL1 was detected, thereby demonstrating the reliability of the screening procedure (Appendix Table S6). Among the eleven additional interacting partners, three proteins belong to the $\mathrm{S}$ AdenosylMethionine (SAM) biosynthesis pathway, the universal methyl group donor (Zhang, 2005). The first one, 5-methylthioribose-1-phosphate isomerase (MTI1, AT2G05830), is involved in the methionine salvage pathway whereas the two others, Methionine Synthase 1 (MS1, AT5G17920) and Methionine Adenosyltransferase 3 (MAT3, AT2G36880) are the ultimate enzymes of the SAM cycle. In order to identify BIN4-interacting proteins in planta, we also performed a ColP-MS experiment using the Arabidopsis mid-1 35S:BIN4/MID-YFP line, which consists of the mid-1 allelic mutant of BIN4 complemented with YFP-tagged BIN4/MID (Kirik et al, 2007), and a wt line as control. To exclude nonspecific proteins, we discarded proteins that were not detected in both BIN4/MID-YFP ColP-MS replicates, as well as chloroplastic, mitochondrial and peroxysomal proteins. The presence of the RHL1 and TOP6B Topo VI subunits in BIN4/MID-YFP ColPs validated our procedure (Appendix Table S7). Remarkably, MAT3 co-immunoprecipitated strongly with BIN4. We further investigated the genetic and biochemical interactions between BIN4 and enzymes of the SAM cycle, particularly the very last enzyme MAT3, using Bimolecular Fluorescence Complementation (BiFC). We confirmed the 
BIN4-MAT3 and BIN4-MS1 interactions in nuclei of transiently agro-transformed Nicotiana benthamiana mesophyll cells (Fig 5A).

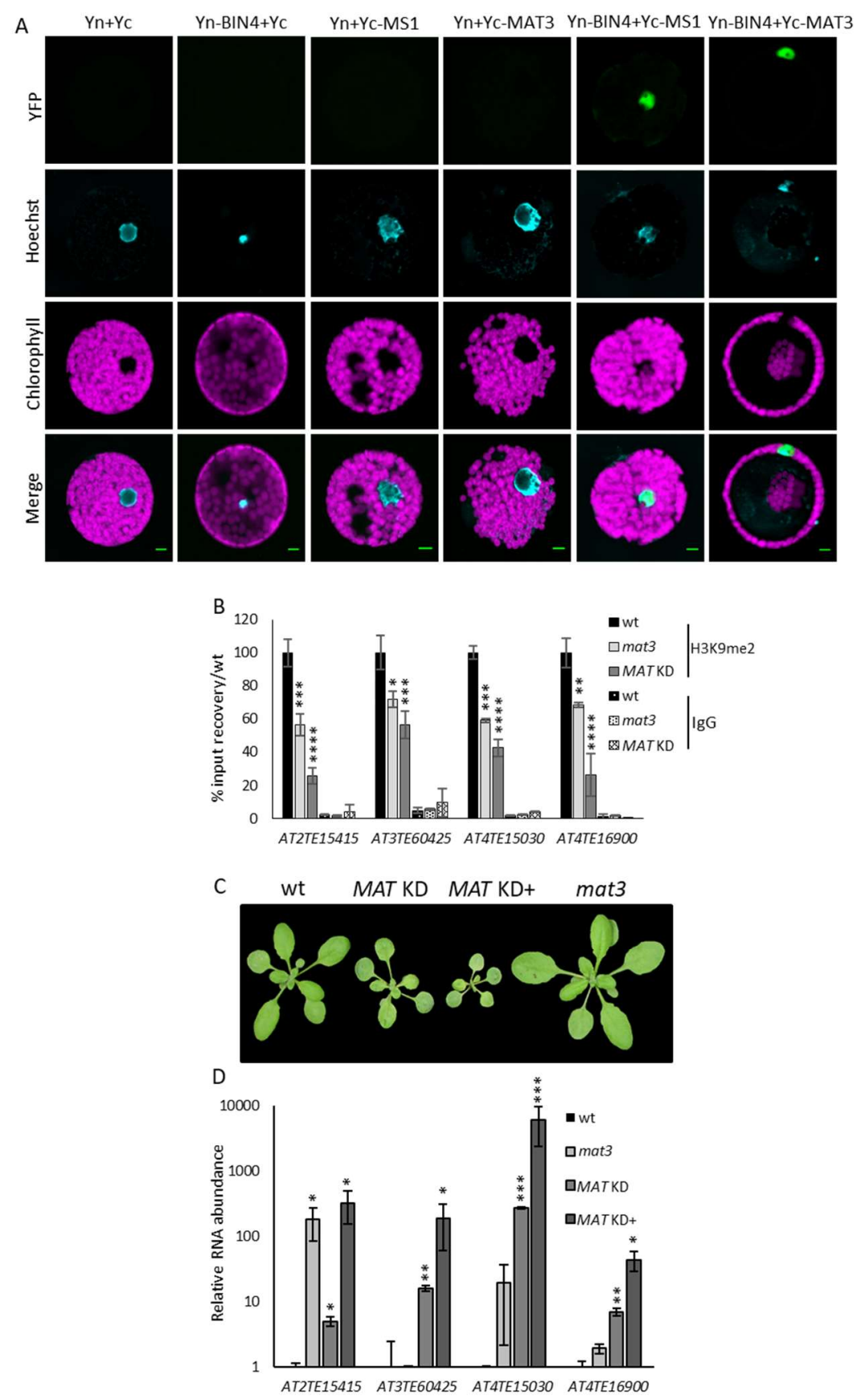

Figure 5. MAT3 directly interacts with Topo $\mathrm{VI}$ and is required for H3K9me2 deposition on heterochromatic loci. (A) Protoplasts from transiently agrotransformed $N$. benthamiana leaves expressing different combination of BiFC vectors, as indicated. Nuclei were stained with Hoechst 33342. (B) Chromatin of 3 week-old wt or MAT KD (here a mixed pool of MATs silenced plants with strong or weak phenotypes) rosette leaves, or 6 day-old wt or mat3 cotyledon nuclei was immunoprecipitated with anti-H3K9me2 antibodies and the recovery of TEs known to be reactivated 
in caa39 was assessed by qPCR. The result is shown as a percentage of recovery normalized against wt. Error bars: \pm SEM of two biological replicates. *: $P<0.05 ;{ }^{* *}: P<0.005 ;{ }^{* *}: P<0.0005 ;{ }^{* * * *}: P<$ 0.0001 (two-way ANOVA, Dunnett's test). (C) Representative photographs of 4 week-old wt, MATs silenced plants with stronger $(M A T K D+)$ and weaker (MAT KD) developmental phenotypes, and the mat3 mutant. (D) RT-qPCR analysis of TE transcript abundance in indicated genotypes. Error bars: \pm SEM of three biological replicates. *: $P<0.05{ }^{* *}: P<0.005{ }^{* * *}$ : $P<0.0005$ (Student's $t$-test).

\section{MAT3 is required for H3K9me2 deposition}

Given that MAT enzymes synthesize the SAM required for DNA and histone methylation, and that Topo $\mathrm{VI}$ is required for proper distribution of $\mathrm{H} 3 \mathrm{~K} 9 \mathrm{me} 2$ throughout El-containing heterochromatic regions, we hypothesized that disruption of MAT3 affects H3K9me2 deposition. To address this question, we used a recently characterized knock-down line in which MAT3 $3^{\prime}$-UTR is interrupted by a T-DNA (Chen et al, 2016), generating strongly reduced but still detectable transcripts levels (Fig S11A). We measured H3K9me2 levels at the four TEs strongly de-repressed in Topo VI mutant plants (Fig 2) by ChIP-qPCR and found decreased levels of H3K9me2 (Fig 5B). This modest decrease of H3K9me2 might be explained by the hypomorphic nature of the mutation or by a functional redundancy between MAT isoforms that share over $85 \%$ amino acid sequence identity (Fig S11B). To test this hypothesis, we took advantage of a homozygous, mono-insertional, co-suppressor transgenic line obtained during the process of generating MAT3-YFP overexpressors, that we referred to as MAT KD (Fig 5C, Fig S11C). Owing to their high DNA sequence identity (Fig S11D), all MAT genes are downregulated in this line (Fig S11E). In addition, the stochastic silencing of MATS gives rise to different phenotype severities: mildly affected MAT KD plants (Fig $5 \mathrm{C}$ ) that accumulate less MATs transcripts than wt (Fig S11E) and present a more severe phenotype than mat3 hypomorphic mutant plants (Fig 5C); and strongly affected MAT KD+ sister plants (Fig 5C) that accumulate even less MAT transcripts (Fig S11E). As anticipated, the H3K9me2 decrease was even more pronounced in MAT KD plants than in mat3 (Fig 5B). These results suggest that MAT isoforms possibly have additive roles in H3K9 dimethylation. Given the decrease of H3K9me2 in MAT-deficient plants, we then determined the extent of TE reactivation in mat3 and MAT KD by RT-qPCR analysis of the same four TEs. We observed increased levels of TE transcripts in mat3 (Fig 5D). This increase was generally more pronounced in MAT KD plants, particularly in MAT KD+ plants (Fig 5D). In contrast, we did not observe any significant effect on El gene expression (Fig S11F).

Topo VI favors MAT enrichment at some heterochromatin borders and depletion from euchromatic islands 
Collectively, our results suggest that Topo VI and MAT3 could act together in maintaining sharp chromatin boundaries by influencing H3K9me2 deposition. We therefore used a newly developed anti-MAT antibody (Agrisera, Vännäs, Sweden) to test for MAT enrichment at specific loci and a putative Topo VI dependency. First, the specificity of this antibody was validated by immunoblot analysis of protein extracts from wt, MAT KD and MAT3-YFP overexpressing lines (Fig S12A). We then performed anti-MATs ChIPs on wt and caa39 plants to measure the recovery of TEs that are reactivated upon Topo VI or MATs loss of functions. Interestingly, MATs were enriched on all the tested TEs in wt and less in caa39, as compared to an IgG negative control (Fig 6A). To specifically evaluate the implication of the BIN4/MID-associated MAT3 isoform, we undertook an anti-GFP ChIP analysis of two independent MAT3-YFP expressing lines. TEs reactivated in MAT KD and in Topo VI mutant plants were also specifically enriched in the GFP-pulled down chromatin (Fig S12B). These results indicate that Topo $\mathrm{VI}$ is required for the association of MAT3 with heterochromatic elements to enable local deposition of H3K9me2.

To test whether Topo VI might also influence the enrichment of MATs at Els, we conducted an anti-MATs ChIP experiment on caa39 and wt plants and probed the same El as in Figures 4E and I, which shows increased H3K9me2 levels within the island body but decreased levels in one heterochromatic border in caa39 (Fig 4E and I, Fig 6B, top panel). We detected a significant decrease of MAT occupancy in this border in caa39 (Fig 6B, bottom panel, probe 1) and, conversely, enhanced MAT occupancy in the island body in caa39 compared to wt (Fig 6B, bottom panel, probes 3-4). We analyzed three other S-Els that show decreased gene expression levels (Fig $3 \mathrm{C}$ ), increased internal H3K9me2 levels (Fig 4C and S8C) and contain a single gene (At4g06634's and At5g30495's Els) or two genes (At2g07340's Els). We generally observed a decrease of MAT occupancy at one or both borders and enhanced MAT occupancy in the island bodies of caa39 (Fig 6C-E). These results show that the loss of Topo VI leads to MAT redistribution over Els, which positively correlates with H3K9me2 redistribution and Els heterochromatinization in caa39. 

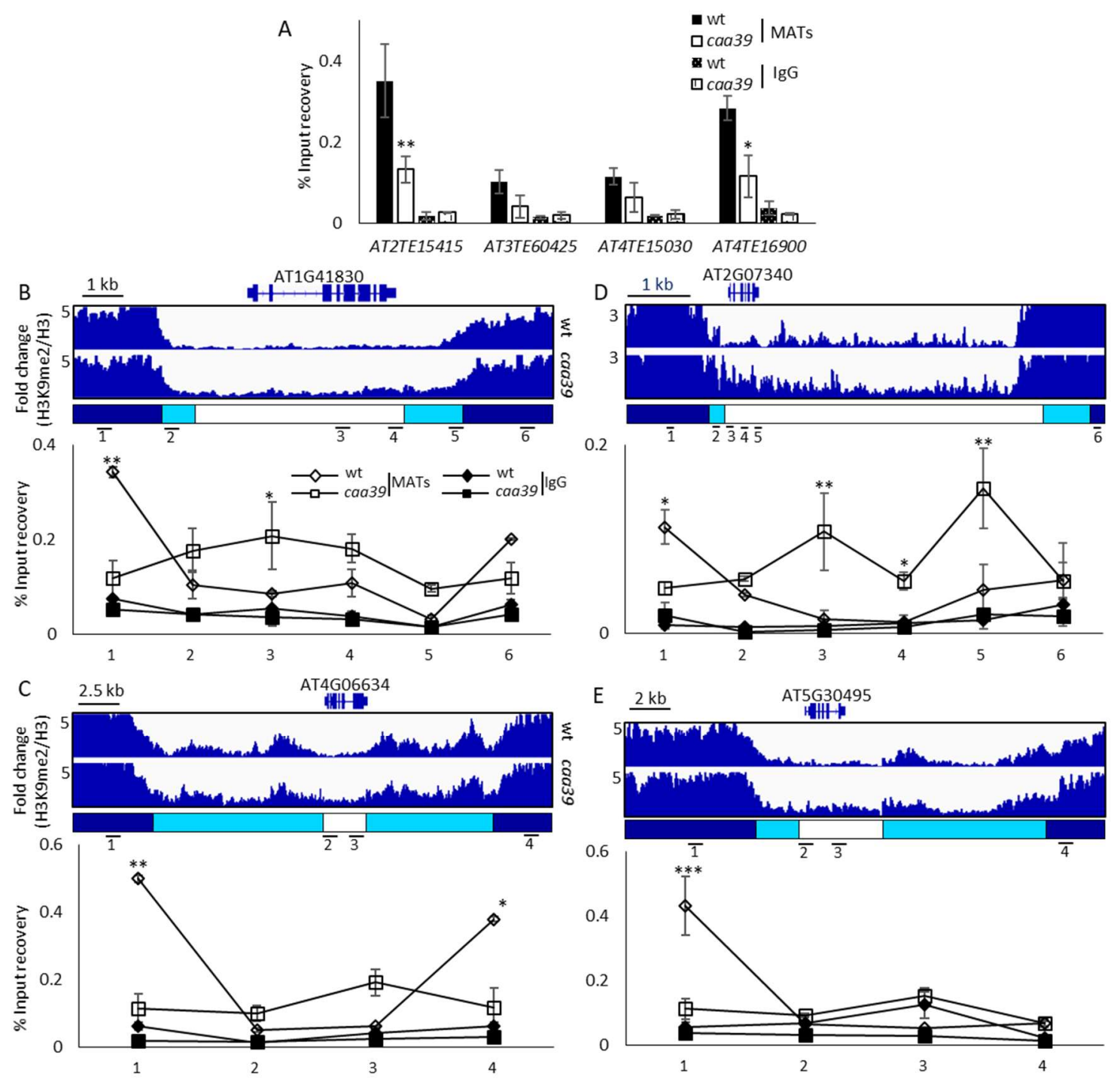

Figure 6. Topo VI is required for MAT enrichment at heterochromatin borders and exclusion from euchromatic islands. (A) Chromatin of 6 day-old wt or caa39 cotyledon nuclei was immunoprecipitated with anti-MATs antibodies and the recovery of TEs reactivated in MATs silenced plants and caa39 was measured by qPCR. Error bars: \pm SEM of three biological replicates. (B-E) Top panels: Integrative Genomics Viewer screenshots of H3- and sequencing depth-normalized H3K9me2 profiles, and locations of primers used in bottom panels. Bottom panels: Same as (A) on Els containing repressed genes in caa39 such as At1g41830 (B), At4g06634 (C), At2g07340 (D) and At5g30495 (E). Error bars: \pm SEM of two biological replicates. *: $P<0.05{ }^{* *}: P<0.005$; $^{* * *}: P<$ 0.0005 (Student's $t$-test). 


\section{Discussion}

The Arabidopsis epigenome is largely indexed by discrete chromatin signatures usually corresponding to single genetic element (e.g., a gene or a TE) (Roudier et al, 2011; Sequeira-Mendes et al, 2014). However, despite this, in Arabidopsis DNA methylation has a known tendency to spread away from many TEs (Ahmed et al, 2011), and a few other studies have reported the existence of heterochromatin spreading in plants (Eichten et al, 2012; Lang et al, 2015; Saze et al, 2008). Yet, the mechanisms that repress heterochromatin spreading, hence safeguarding Els, are poorly understood in plants. Our study confirmed the existence of an insulator-like mechanism that preserves Els and unveiled the role played by the Topo VI complex in this process. We first provide evidence that Topo $\mathrm{VI}$ is required to preserve the euchromatic nature and transcriptional activity of gene islands within pericentromeric and chromosome 4 knob heterochromatic regions. Indeed, the most remarkable effect of the caa39 mutation was the specific misregulation of pericentromeric elements, with a general downregulation of El genes and, inversely, a reactivation of heterochromatic TEs. We confirmed this peculiar expression pattern in several amorphic and hypomorphic mutants of the Topo VI complex. Surprisingly, El gene downregulation is more pronounced in the hypomorphic mutants caa39 and BIN4 KD than in the corresponding null Topo VI mutants that display more severe growth defects. Taking advantage of uncoupled growth defects and gene expression changes in the hypomorphic caa39 allele, we were able to show that the repression of El genes is correlated with the invasion of Els by H3K9me2, indicating that Topo $\mathrm{VI}$ is required for preventing H3K9me2 spreading, here referred to as a boundary function. The level of H3K9me2 in Els of caa39 remain, however, significantly lower than in canonical heterochromatin. This might result from histone H3 demethylation, a process that is likely still active in caa39 as we did not observe any alteration of expression of key demethylation genes such as IBM1. Alternatively, gain of H3K9me2 could be the outcome of a subset of cells in which caa39 mutation has a strong effect on H3K9me2 boundaries.

Unexpectedly, WGBS identified no global increase of cytosine methylation over Els, but rather a decrease of CHG methylation, despite CHGs being known to be methylated through a feedback loop with H3K9me2. Recent studies report that, under specific circumstances, increased H3K9me2 levels do not necessarily induce increased $\mathrm{CHG}$ or $\mathrm{CHH}$ methylation, and vice versa. For instance, the AT-hook protein AHL10 ectopically recruits H3K9me2 to small, AT-rich TEs without coincidental increases in DNA methylation (Jiang et al, 2017). Another study reports that expression of AtCMT3 in Eutrema Salsugineum, a Brassicaceae that has lost CMT3 and gene body methylation, indeed induce de novo gene body methylation in CHG, $\mathrm{CHH}$ and CG contexts, but does not induce gains in H3K9me2. Interestingly, hyper-CHG methylation in gene bodies was not correlated with consistent changes in gene expression (Wendte et al, 2019). More generally, the interplay between 
H3K9me2 and DNA methylation might be regulated by the kinetics of activity of both pathways. Indeed, heterochromatin is known to inhibit and exclude RdDM (Zemach et al, 2013; Schoft et al, 2009; Gent et al, 2014). Thus, it is plausible that H3K9me2 spreading over El genes is sufficient to induce transcriptional silencing without the involvement of DNA methylation in a context of lack of Topo VI. Nonetheless, we have no indication to rule out a possible role of DNA methylation and/or ncRNAs in the delineation of sharp chromatin boundaries as Els. Interestingly, RNA Pol V-produced ncRNAs also play a role in the determination of heterochromatic identity in Arabidopsis but are very unlikely involved in the inhibition of heterochromatin spreading (Böhmdorfer et al, 2016). Inversely, RNA-directed DNA methylation (RdDM) appears to be required to inhibit the spreading of euchromatin into heterochromatin in maize (Li et al, 2015). Nevertheless, TEs can provide insulator sequences in animals (Wang et al, 2015b) and the activity of CTCF is known to be regulated by ncRNAs (Cohen \& Jia, 2015). In fission yeast, the non-coding RNA (ncRNA) BORDERLINE prevents the spreading of heterochromatin to euchromatin by evicting the H3K9me reader and chromatin barrier protein Swi6/HP1 from euchromatin (Keller et al, 2013).

The disorganization of chromocenters and the loss of pericentromeric TE silencing observed in Topo VI mutants might result from the loss of this boundary function, and likely also by the loss of an distinct architectural function of Topo VI in heterochromatin condensation. Indeed, given that no obvious loss of DNA methylation and only a partial decrease of H3K9me2 over TEs were observed in Topo VI mutant plants, the reactivation of TEs is unlikely to be solely explained by decreased levels of these marks, but rather by a combined loosening of their heterochromatic nature and of their higher order organization. It is worth noting that in animals, insulator proteins can participate in several distinct processes. For instance CTCF acts locally as a chromatin barrier and more globally on the formation of topologically associating domains (Lu et al, 2016). Furthermore, the co-occurrence of EI invasion by $\mathrm{H} 3 \mathrm{~K} 9 \mathrm{me} 2$ and the increase of $\mathrm{H} 3 \mathrm{~K} 27 \mathrm{me} 3$ within heterochromatin supports a dual breaking model of the Arabidopsis Topo VI boundary function. Alternatively, heterochromatin spreading might itself perturb H3K27me3 deposition by PRC2 and/or erasing by trithorax group (trxG) proteins. This second hypothesis is supported by similar observations made in other organisms, where the loss of Swi6/HP1 leads to H3K9me2 spreading across natural constitutive heterochromatin boundaries in fission yeast (Stunnenberg et al, 2015), and alters H3K27me3 deposition on facultative heterochromatin in Neurospora crassa (Jamieson et al, 2016).

Notably, other plant GHKL ATPases such as the MORC proteins are also required for TE silencing and chromocenter formation without strongly impacting H3K9me2 and DNA methylation levels (Moissiard et al, 2012; Lorkovi et al, 2012; Brabbs et al, 2013). Although atmorc1 and atmorc6 mutants appear to be less dramatically affected than caa39, Topo VI might act downstream of DNA 
methylation in a similar manner to what Moissiard et al. reported for MORC proteins (Moissiard et al, 2012). Interestingly, human CTCF has been shown to interact with Topo $I \beta$ (Witcher \& Emerson, 2009; Yusufzai et al, 2004) and appears to be part of a protein interaction network that also contains MORC2 and members of the cohesin complex in Hela cells (Uusküla-Reimand et al, 2016). Intriguingly, plant and human recombinant MORC proteins seem to display a type II topoisomeraselike activity, which requires additional factors for full activity. However, the possibility that topoisomerases co-purify with MORC proteins cannot be completely ruled out (Manohar et al, 2017). Collectively, our study on plant Topo VI extends the role of topoisomerase function to chromatin, which is an emerging theme in a wide range of organisms.

Although we cannot rule out the implication of other MATs in the definition of boundaries and/or El identity, the finding that the Topo VI BIN4 subunit directly interacts with MAT3 and is required for MAT3 enrichment at TE loci and depletion from euchromatic islands, argues for a local role of MAT3 on chromatin, in addition to its general role in SAM synthesis. Interestingly, a homologue of MAT3 in mammals, MATIla, also directly supplies SAM in the close vicinity of oncogenes to allow transcriptional repression and H3K9me2 deposition (Katoh et al, 2011; Kera et al, 2013). The mouse MATIl $\alpha$ has further been found to interact with Topoisomerase Il $\alpha$, a type IIA topoisomerase whose C-terminal regulatory domain possesses sequence similarity with the BIN4 subunit of the plant Topo VI complex (Breuer et al, 2007). Hence, although this requires testing in other organisms, an interaction between MAT enzymes and type II topoisomerases might be evolutionary conserved. Targeting of MATs to specific chromatin regions by topoisomerases might be a way to couple SAM synthesis and availability in situ, possibly for DNA or histone methylation. An analogous system might also exist between MAT, histone methyltransferases and type I topoisomerases in plants. Indeed, Xuemei Chen's group has recently reported that Topo I $\alpha$, in addition to being involved in TE silencing through RdDM and H3K9 dimethylation (Dinh et al, 2014), is also required for Polycomb-mediated gene regulation (Liu et al, 2014). Interestingly, by mining mass spectrometry data of immuno-purified CLF and ALP1 PCG subunits (Liang et al, 2015), residual levels of MAT3 and more abundant levels of MAT4 could be identified (data not shown). In plants, the existence of an insulator-like function that would partition chromatin into different functional domains has long been questioned (Wang et al, 2015a; Liu et al, 2017; Vergara \& Gutierrez, 2017). We show that Topo VI participates to such a function by preventing the spreading of the heterochromatic mark $\mathrm{H} 3 \mathrm{~K} 9 \mathrm{me} 2$ into neighboring euchromatin islands. Our results suggest that the prevention of heterochromatin spreading relies upon Topo VI-dependent targeting of MAT3 to heterochromatin, and exclusion from euchromatic islands. Future studies might allow the identification of direct molecular links between MAT proteins and methyltransferases involved in 
DNA, H3K9 and/or H3K27 methylation for fine-tuning the establishment of sharp transitions in chromatin identity along the genome.

\section{Materials and Methods}

\section{Cloning and plasmids}

cDNA containing the full-length BIN4.3 (AT5G24630.3), MAT3 (AT2G36880) and MS1 (AT5G17920) open reading frames were amplified by RT-PCR from Arabidopsis thaliana ecotype Col-0 and with primers listed in Additional file 8. The PCR products were introduced by BP recombination into the pDONR207 entry vector (Invitrogen) and sequenced to ensure the absence of mutations. These entry clones, with or without a stop codon, were then introduced into destination vectors using LR recombination and sequenced again to confirm that the fusions were in frame. To generate transgenic lines, the entry clones were transferred to pEarleyGate 102 (BIN4-CFP-HA) or pEarleyGate 101 (MAT3-YFP-HA) binary vectors (Earley et al, 2006). For BiFC assays, the entry clones were transferred to the four pBiFP vectors (Azimzadeh et al, 2008). All binary vectors for transient or stable plant transformation were then transferred to Agrobacterium tumefaciens strain C58C1 RifR (pMP90) by electroporation.

\section{Plant material and growth conditions}

All lines are in the Col-0 ecotype. ddm1-8 (SALK_000590C) was ordered from the NASC collection. The rhl2-1, bin4-1, caa39 and mat3 mutants are already described (Breuer et al, 2007; Chen et al, 2016; Simkova et al, 2012; Sugimoto-Shirasu et al, 2002). The MAT3-YFP and BIN4-CFP-HA transgenic lines were generated using the floral dip method (Zhang et al, 2006) and single insertion homozygous lines were selected. In all experiments except those using the MAT KD line, plants were cultivated on half strength MS/Agar medium with $16 \mathrm{~h}$ light/ $8 \mathrm{~h}$ dark cycles for six days at $80-90 \mu \mathrm{mol}$ photons $\mathrm{m}^{-2}$ $\mathrm{s}^{-1}$ light intensity. For other experiments, plants were cultivated in soil for three to four weeks under the same light regime as stated above.

\section{DNA preparation, Chop-qPCR and Anti-5-meC ELISA assay}

Genomic DNA was prepared using the NucleoSpin ${ }^{\circledast}$ Plant II Midi kit (Macherey-Nagel). For each reaction (either with McrBC, Hpall, Mspl or HaellI), 200 ng DNA was used in a $20 \mu \mathrm{L}$ final reaction volume. 5 units of Mspl, Hpall or Haelll per 100 ng DNA were used during $1 \mathrm{~h}$ at $37^{\circ} \mathrm{C}$. For McrBC 
digestion, 1 unit per $100 \mathrm{ng}$ DNA was used for an overnight digestion at $37^{\circ} \mathrm{C}$. One nanogram of undigested or digested samples was used for qPCR. Undigested samples were treated the same temperature and for the same time but without enzyme. The result is expressed as $2^{-\mathrm{Cl}_{\mathrm{t}}}$ digested ${ }_{\text {undigested. }}^{\mathrm{C}^{\prime}}$. Global methylation of cytosines was assessed using the MethylFlash global DNA methylation ELISA easy kit following the manufacturer's instructions (Epigentek).

\section{Yeast Two-hybrid Screen}

The yeast two-hybrid screen was performed by Hybrigenics using the Arabidopsis RP1 library. The full-length BIN4 cDNA (AT5G24630.3/4) was used as bait.

\section{Co-immunoprecipitation-MS}

$500 \mathrm{mg}$ of ten-day-old wt and mid1 MID-YFP seedlings were harvested and ground in liquid nitrogen, mixed with $2 \mathrm{~mL}$ of RIPA buffer (10 mM TrisHCl pH 7.5, $150 \mathrm{mM} \mathrm{NaCl}, 0.5 \mathrm{mM}$ EDTA, $0.1 \%$ SDS, $1 \%$ Triton X-100, 1\% sodium deoxycholate, protease inhibitors). Samples were incubated for $30 \mathrm{~min}$ in ice and gently resuspended every $10 \mathrm{~min}$, centrifuged for $15 \mathrm{~min}, 16,100 \times \mathrm{g}, 4{ }^{\circ} \mathrm{C}$. For bead preparation, rabbit monoclonal anti-BIN4 (Breuer et al, 2007) and rabbit polyclonal anti-GFP antibodies (Thermo-Fisher, Cat. No. A-11122) were added in equal quantity (16 $\mu \mathrm{g}$ each) to $80 \mu \mathrm{L}$ of Dynabeads ${ }^{\circledR}$ protein A (Thermo-Fisher, Cat. No. 10001D). Antibodies were incubated with beads overnight at $4{ }^{\circ} \mathrm{C}$ before crosslinking with DMP following the manufacturer's protocol (Abcam). Then, antibody-conjugated beads were incubated with protein extracts for $2 \mathrm{~h}$ at $4{ }^{\circ} \mathrm{C}$, and washed three times in immunoprecipitation buffer (1 mM EDTA, 10\% glycerol, $75 \mathrm{mM} \mathrm{NaCl}, 0.05 \%$ SDS, $100 \mathrm{mM}$ TrisHCl pH 7.4, $0.1 \%$ Triton X-100). Proteins were eluted by incubating at $70^{\circ} \mathrm{C}$ in Laemmli buffer and the eluates were briefly migrated on SDS-PAGE before MS analysis.

\section{Western blot}

For nuclear proteins, nuclei were prepared as described in the ChIP protocol below. The nuclei pellet was resuspended in nuclei lysis buffer and proteins were quantified using the bicinchoninic acid assay. $60 \mu \mathrm{g}$ of nuclear proteins was separated on 12\% SDS-PAGE and transferred to PVDF membranes. For total protein extracts, tissue powder was resuspended in SDS loading buffer $(\mathrm{v} / \mathrm{v})$, centrifuged $16,100 \times \mathrm{g}, 10 \mathrm{~min}, 4{ }^{\circ} \mathrm{C}$, and boiled $5 \mathrm{~min}$. BIN4, H3K9me2 (Abcam ab1220, lot: 
GR244373-6), H3 (Abcam ab1791, lot: GR178101-1), MAT (Agrisera AS16 3148) and GFP (Roche 11814460001, lot: 12600500) antibodies were diluted 1:3000, 1:2000, 1:1000, 1:5000, 1:3000 and 1:3000 in TBST/milk 0.5\%, respectively. After blocking $1 \mathrm{~h}$ in $5 \%$ milk, membranes were incubated with the primary antibody for $1 \mathrm{~h}$, washed 5 times with TBST and incubated with secondary HRPcoupled anti-mouse or anti-rabbit antibodies at 1:20000 during $1 \mathrm{~h}$. Membranes were washed 5 times and revealed with Immobilon western chemiluminescent HRP substrate (Millipore).

\section{RNA extraction, RT-qPCR and microarrays}

RNA was extracted using Trizol following the manufacturer's instructions. The integrity of RNA was verified by migrating $1 \mu \mathrm{g}$ RNA on a 1.5\% agarose gel. Then, 500 ng RNA was DNAse l-treated (ThermoFischer Scientific) and submitted to reverse transcription (Takara PrimeScript ${ }^{\mathrm{TM}}$ RT). Before qPCR, the absence of genomic DNA and equal RNA loading per sample was verified using semiquantitative RT-PCR against ACT2 (see Appendix Table S8 for a complete primer list). cDNAs were diluted twice and $1 \mu \mathrm{L}$ was used per qPCR reaction in a final volume of $15 \mu \mathrm{L}$. PP2A (AT1G13320) and PRF1 (AT2G19760) were used as control genes in every RT-qPCR experiment. Affymetrix Arabidopsis ATH1 GeneChips were used to analyse bin4-1 and bin4-2 mutant lines. Raw data processing was performed using the RMAExpress software (http://rmaexpress.bmbolstad.com/). Data were normalized by using background adjusting and quantile normalization.

\section{RNA-seq library preparation and sequencing}

Three independent biological replicates were produced. For each biological repetition, RNA samples were obtained by pooling RNAs from more than 100 plants. Aerials parts were collected on plants at 1.00 developmental growth stages (Boyes et al, 2001), cultivated as described above. Total RNA was extracted using RNeasy kit (Qiagen ${ }^{\circledR}$, Hilden, Germany) according to the supplier's instructions. RNAseq experiment was carried out at plateform POPS, transcriptOmic Plateform of institute of Plant Sciences - Paris-Saclay, thanks to IG-CNS Illumina Hiseq2000 privileged access to perform paired-end 100bp sequencing, on RNA-seq libraries constructed with the TruSeq_Stranded_mRNA_SamplePrep_Guide_15031047_D protocol (Illumina ${ }^{\circledR}$, California, U.S.A.). The RNA-seq samples have been sequenced in paired-end (PE) with a sizing of $260 \mathrm{bp}$ and a read length of 100 bases. Six samples by lane of Hiseq2000 using individual bar-coded adapters and giving approximately 30 million of PE reads by sample were generated.

\section{RNA-seq bioinformatic treatment and analysis}


To facilitate comparisons, each RNA-Seq sample followed the same workflow from trimming to transcript abundance quantification, as follows. Read preprocessing criteria included trimming of library adapters and performing quality control checks using FastQC (v0.10.1). The raw data (fastq) were trimmed for Phred Quality Score $>20$, read length $>30$ bases and sort by Trimming Modified homemade fastx_toolkit-0.0.13.2 software for rRNA sequences. Bowtie v2 (Langmead \& Salzberg, 2012) was used to align reads against the Arabidopsis thaliana transcriptome (with --local option). The 33602 mRNAs were extracted from TAIRv10 database (Lamesch et al, 2012) with one isoform per gene to avoid multi-hits. The abundance of mRNAs was calculated by a local script which parses SAM files and counts only paired-end reads for which both reads map unambiguously to the same gene. According to these rules, around $98 \%$ of PE reads aligned to transcripts for each sample. Gene expression analysis was performed using likelihood ratio test for a negative binomial generalized linear model where the dispersion was estimated by the edgeR method (v1.12.0, (McCarthy et al, 2012)) in the statistical software ' $R$ ' (v2.15.0, (R Development Core Team, 2017)). TEtranscripts (Jin et $a l, 2015)$ and edgeR were used to discover differentially expressed transposable elements in caa39 compared to wt. The reference genome was Arabidopsis_thaliana TAIR10.31.gtf and the TE reference was TAIR10_TE.gtf. TEtranscripts options: multi-mapper mode and SAM files.

\section{Whole-genome bisulfite sequencing and DNA methylation analysis}

Seeds of wt and caa39 were sowed with an interval of one week to generate biological replicates. For each replicate, DNA samples were obtained from $400 \mathrm{mg}$ of fresh aerial parts of 6 day-old plants (developmental growth stages (Boyes et al, 2001)) crushed in liquid nitrogen. Genomic DNA was prepared using the NucleoSpin ${ }^{\circledast}$ Plant II Midi kit (Macherey-Nagel), with the addition of Poly(vinylpyrrolidone) to the powdered sample. Libraries were prepared and sequenced at the BGI (China). Quality control was performed on sequencing files using FASTQC (v0.11.5) and adapter trimming was performed at BGI. Methylation analysis has been performed with Bismark (v0.20.0) (Krueger \& Andrews, 2011) using default parameters, except -CX option, to get data for all the three contexts ( $\mathrm{CG}, \mathrm{CHH}$ and $\mathrm{CHG}$ ). The bedgraph output file has been filtered into 3 files, one for each context. DMRfinder (Gaspar \& Hart, 2017) was used to identify differentially methylated regions and the "combine_CpG_sites.py" script was used to cluster CpG (and other context) sites into regions with data_bismark.cov files as input files and default parameters. findDMRs.r was used with default parameters to conduct pairwise tests of sample groups in order to identify DMRs.

\section{Chromatin Immunoprecipitation}


Two hundred 6-day-old seedlings were used per biological replicates. Seedlings were quickly harvested using a cat hair comb, root material was cut-off and the remaining photosynthetic material was fixed with $1 \%$ formaldehyde for $10 \mathrm{~min}$. Crosslinking was quenched by addition of glycine at 125 $\mathrm{mM}$ final concentration and 5 min incubation, washed twice in $\mathrm{dd}_{2} \mathrm{O}$ and the excess water was drained using kimwipes. Plant material was then ground in liquid nitrogen, resuspended in extraction buffer 1 (0.4 M Sucrose, $10 \mathrm{mM}$ Tris $\mathrm{pH} 8,10 \mathrm{mM} \mathrm{MgCl}$, $5 \mathrm{mM} \beta$-mercaptoethanol and protease inhibitor), filtered with four layers of miracloth and centrifuged $20 \mathrm{~min}$ at $3,300 \times \mathrm{g}, 4^{\circ} \mathrm{C}$. The pellet was resuspended in extraction buffer 2 (0.25 M Sucrose, 1\% Triton X100, $10 \mathrm{mM}$ Tris pH 8, $10 \mathrm{mM}$ $\mathrm{MgCl}_{2}, 5 \mathrm{mM} \beta$-mercaptoethanol and protease inhibitor) and centrifuged $10 \mathrm{~min}, 16,100 \times \mathrm{g}, 4{ }^{\circ} \mathrm{C}$. The pellet was resuspended in $300 \mu \mathrm{L}$ extraction buffer 2 and layered on top of $300 \mu \mathrm{L}$ extraction buffer 3 (1.75 M Sucrose, 0.15\% Triton X100, $10 \mathrm{mM}$ Tris $\mathrm{pH} 8,2 \mathrm{mM} \mathrm{MgCl}, 5 \mathrm{mM} \beta$ mercaptoethanol and protease inhibitor) and centrifuged at $16,100 \times g$ for $1 \mathrm{~h}, 4^{\circ} \mathrm{C}$. The resulting pellet was resuspended in $130 \mu \mathrm{L}$ of nuclear lysis buffer $(50 \mathrm{mM}$ Tris pH 8, $10 \mathrm{mM}$ EDTA, 1\% SDS and protease inhibitor) and sheared using a Bioruptor for 10 cycles, high settings, $30 \mathrm{sec}$ ON/60 sec OFF, twice. Then, the sheared chromatin was diluted ten times in ChIP dilution buffer (1.1\% Triton X100, $16.7 \mathrm{mM}$ Tris $\mathrm{pH}$ 8, $1.2 \mathrm{mM}$ EDTA, $167 \mathrm{mM} \mathrm{NaCl}$ and protease inhibitor). In parallel, $10 \mu \mathrm{L}$ protein $\mathrm{G}$ Dynabeads ${ }^{\circledR}$ were washed twice in ChIP dilution buffer and incubated either with $2 \mu \mathrm{g}$ of mouse IgG (Sigma), anti-H3K27me3 (Abcam ab6002, lot: GR275911-2), anti-H3K9me2, anti-MAT or anti-GFP during $2 \mathrm{~h}$ at $4{ }^{\circ} \mathrm{C}$ under gentle agitation. Antibody-conjugated beads were washed twice with $100 \mu \mathrm{L}$ ChIP dilution buffer and $400 \mu \mathrm{L}$ of input was submitted to IP for $4 \mathrm{~h}$ or at $4{ }^{\circ} \mathrm{C}$ under gentle agitation. Immunocomplexes were washed twice with each following buffer, $5 \mathrm{~min}, 4^{\circ} \mathrm{C}$, gentle agitation, in that order: low salt wash buffer (150 mM NaCl, 0.1\% SDS, 1\% Triton X100, 2 mM EDTA, 20 mM Tris $\mathrm{pH}$ 8), high salt wash buffer ( $500 \mathrm{mM} \mathrm{NaCl}, 0.1 \%$ SDS, $1 \%$ Triton X100, 2 mM EDTA, $20 \mathrm{mM}$ Tris pH 8), $\mathrm{LiCl}$ wash buffer (250 mM LiCl, 1\% Igepal CA-630, 1\% sodium deoxycholate, 1 mM EDTA, 10 mM Tris $\mathrm{pH}$ 8), TE buffer (10 mM Tris pH 8, 1 mM EDTA). Immunocomplexes were then eluted twice with 100 $\mu \mathrm{L}$ elution buffer (100 mM NaHCO $3,1 \% \mathrm{SDS}$ ), $15 \mathrm{~min}$ at $65{ }^{\circ} \mathrm{C}$ and agitation. The $200 \mu \mathrm{L}$ eluate was reversed crosslinked by adding $16 \mu \mathrm{L}$ of $2.5 \mathrm{M} \mathrm{NaCl}$ and incubating at $65^{\circ} \mathrm{C}$ overnight and DNA was purified using phenol/chloroform extraction after RNase A and proteinase K treatment. A $40 \mu \mathrm{L}$ aliquot of input was reversed crosslinked and purified in parallel. DNA was quantified using Qubit ${ }^{\circledR}$ dsDNA HS assay kit (ThermoFischer Scientific) and equal amounts of input or IPed DNA were used per qPCR reaction.

\section{ChIP-seq analysis}


Each ChIP biological replicate was prepared by pooling two independent IP eluates. ChIP-seq librairies from two independent biological replicates of H3K27me3, H3 and inputs and three independent biological replicates of H3K9me2, were generated and sequenced on a NextSeq 500 Illumina at the University of Aix-Marseille TAGC U1090 platform. Sequence quality was checked using FASTQC (v0.11.5) (Andrews, 2010). Sequences were trimmed with Trimmomatic (v0.36) (Bolger et al, 2014) with the following options : ILLUMINACLIP:TruSeq3-SE.fa with 1 mismatch allowed, and MINLEN:65. Reads were then aligned to the genome using Bowtie v2 (v2.2.9) (Langmead \& Salzberg, 2012). The output Sam files were converted to bam with samtools (v1.3.1) (samtools view -bt) using Arabidopsis_thaliana TAIR10.31.dna.genome.fa as a reference. The reads with poor mapping quality were removed with bamtools (v2.4.0) (Barnett et al, 2011) and reads with a MAPQ>=20 were kept. The PCR duplicates were removed with the MarkDuplicates tool (v2.8.1) with default options. Peak calling was performed using the diffReps-nb tool (v1.55.2) and bam files were converted to bed files using bedtools (v2.25.0). Peak calling was performed with a window of 200, $p$-value of 0.01 and $g$ test (individual replicate analysis) or negative binomial (analysis with all replicates, Appendix Table S5) statistical tests. The H3 samples of Col-0 and caa39 were used as background. Annotation was made using PAVIS (Huang et al, 2013). For visualization of the data with IGV (v2.3.88) (Robinson et al, 2011; Thorvaldsdóttir et al, 2013), bigwig files were generated from the alignment files with BamCoverage (v2.4.1) (Ramírez et al, 2014). Bam files were indexed with samtools index and then the bigwig files were generated with bamCoverage using the -normalizeTo1x option. The linear Kmeans clustering method implemented in seqMINER v1.3.4 (Ye et al, 2011) was used.

\section{Extraction of euchromatic island sequences}

Those islands harbor characteristic chromatin states of expressed gene and are surrounded by state 9 and 8. Using a homemade perl script, we screened for all regions within heterochromatic regions that are surrounded by chromatin state 8 in the proximal region and state 9 in the distal region, as defined by Sequeira-Mendes et al., 2014. The length threshold of state 9 was set at $450 \mathrm{bp}$ and of the state $8,150 \mathrm{bp}$. The list of coordinates generated by this script was then converted to a bed file and used to define the region of interest in the bigwig files. In order to map read density along islands, we used the aggregate command of the bwtool (v1.0) (Pohl \& Beato, 2014) with the functionality up:meta:down where meta is set to the median length (in bp) of the element studied.

\section{Statistical analysis of the Eukaryotic island profiles}


The matrix program of Bwtools (https://github.com/CRG-Barcelona/bwtool/wiki/matrix) was used to extract the data from bigwig files. Using the up:meta:down option, the data was extracted in the same way as the aggregate program but instead of returning a mean for each position, it creates a matrix containing each replicate value for each position. The H3K9me2 or H3K27me3 matrix were normalized by the $\mathrm{H} 3$ matrix, replicates were concatenated in one matrix which was then treated as the aggregate file. A Shapiro test was first performed to check for normal distribution. The nonparametric Mann-Whitney test was used to compare the value for WT and caa39 at each position.

\section{Immunofluorescence}

Cytological experiments were conducted as previously described (Bourbousse et al, 2015) with a minor modification. For immunolocalization of methylated histones, forty cotyledons were extensively chopped in Galbraith buffer ( $45 \mathrm{mM} \mathrm{MgCl}_{2}, 20 \mathrm{mM}$ MOPS, $30 \mathrm{mM}$ sodium citrate, $0.3 \%$ triton X-100, pH7). Anti-5-methylcytosine (Eurogentec BI-MECY-0100, lot: vt150601) or antiH3K9me2 (Abcam ab1220, lot: GR244373-6) antibodies were used at 1:100. Anti-mouse antibodies coupled with Alexa 488 (ab150125, lot: GR285477-1) were used at 1:200. At least 15 individual nuclei were observed in each condition and experiments were independently repeated twice.

\section{Transient transformation and protoplasts preparation}

Agrobacterium-mediated transient transformation of Nicotiana benthamiana was done as previously described (Meteignier et al, 2016) with minor modification. Overnight cultures of bacteria were

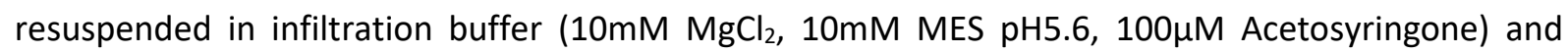
incubated $2 \mathrm{~h}$ before infiltration. P19 was added in every infiltration combination. Protoplasts were prepared as previously described (Yoo et al, 2007) with minor modifications: enzymes solution was not filtered and protoplasts were not washed.

\section{Confocal and epifluorescence microscopy}

Images were taken on a Zeiss LSM 780 confocal microscope for immunofluorescence experiments or an Axiolmager Z1 apotome for every other experiment. All confocal images were acquired using identical parameters. 
Availability of data and material

ChIP-seq, RNA-seq, BS-seq and microarray datasets are available at https://www.ncbi.nlm.nih.gov/geo/query/acc.cgi?acc=GSE103924

\section{Acknowledgments}

We thank Imen Mestiri (IBENS, Paris) for her expertise with cytogenetics. We also want to express our gratitude to students who contributed to this work, especially Justine Quillet, Julien Vieu and César Botella. We thank Ben Field for critical reading of the manuscript. This work was supported by the French National Research Agency (ANR 2010-JCJC-1205-01 and ANR-14-CE02-0010 to CL). Work by FB was supported by the Investissements d'Avenir LabexMemory in Living Systems (MEMOLIFE) grant ANR-10-LABX-54. LD was supported by CEA and Région PACA. High-throughput RNAsequencing was performed at the POPS plateform, supported by the LabEx Saclay Plant Sciences-SPS (ANR-10-LABX-0040-SPS). MS analysis was performed at the IMM platform supported by a grant from GIS IBiSA. High-throughput ChIP-seq was performed at the TGML platform, supported by grants from Inserm, GIS IBiSA, Aix-Marseille Université, and ANR-10-INBS-0009-10.

\section{Author contributions}

LVM, FV, CV, LD, MR, and CB performed the experiments. $C L$ and $M T$ performed the bioinformatic analyses. LST was in charge of the sequencing. LVM, CL, FB and CL analyzed the data. LVM, KS, FB and $\mathrm{CL}$ designed the research. $\mathrm{LVM}, \mathrm{FB}$ and $\mathrm{CL}$ wrote the manuscript. All authors read and approved the final manuscript.

\section{Conflict of interest}

The authors declare that they have no conflict of interest

\section{References}

Ahmed I, Sarazin A, Bowler C, Colot V \& Quesneville H (2011) Genome-wide evidence for local DNA methylation spreading from small RNA-targeted sequences in Arabidopsis. Nucleic Acids Res.

39: 6919-6931 
Ali T, Renkawitz R \& Bartkuhn M (2016) Insulators and domains of gene expression. Curr. Opin. Genet. Dev. 37: 17-26

Andrews S (2010) FastQC: A quality control tool for high throughput sequence data. : http://www.bioinformatics.babraham.ac.uk/projects/

Azimzadeh J, Nacry P, Christodoulidou A, Drevensek S, Camilleri C, Amiour N, Parcy F, Pastuglia M \& Bouchez D (2008) Arabidopsis TONNEAU1 proteins are essential for preprophase band formation and interact with centrin. Plant Cell 20: 2146-2159

Barnett DW, Garrison EK, Quinlan AR, Str̈mberg MP \& Marth GT (2011) Bamtools: A C++ API and toolkit for analyzing and managing BAM files. Bioinformatics 27: 1691-1692

Bergerat A, Gadelle D \& Forterre P (1994) Purification of a DNA topoisomerase II from the hyperthermophilic archaeon Sulfolobus shibatae. A thermostable enzyme with both bacterial and eucaryal features. J. Biol. Chem. 269: 27663-9

Bergerat A, de Massy B, Gadelle D, Varoutas P-C, Nicolas A \& Forterre P (1997) An atypical topoisomerase II from archaea with implications for meiotic recombination. Nature 386: 414417

Bickmore WA \& van Steensel B (2013) Genome Architecture: Domain Organization of Interphase Chromosomes. Cell 152: 1270-1284

Böhmdorfer G, Sethuraman S, Rowley MJ, Krzyszton M, Rothi MH, Bouzit L \& Wierzbicki AT (2016) Long non-coding RNA produced by RNA polymerase $V$ determines boundaries of heterochromatin. Elife 5: 1-24

Bolger AM, Lohse M \& Usadel B (2014) Trimmomatic: a flexible trimmer for Illumina sequence data. Bioinformatics 30: 2114-2120

Bonev B \& Cavalli G (2016) Organization and function of the 3D genome. Nat. Rev. Genet. 17: 772772

Bourbousse C, Mestiri I, Zabulon G, Bourge M, Formiggini F, Koini MA, Brown SC, Fransz P, Barneche F, Bowler C \& Barneche F (2015) Light signaling controls nuclear architecture reorganization during seedling establishment. Proc. Natl. Acad. Sci. 112: 2836-2844

Boyes DC, Zayed AM, Ascenzi R, McCaskill AJ, Hoffman NE, Davis KR \& Görlach J (2001) Growth stagebased phenotypic analysis of Arabidopsis: a model for high throughput functional genomics in plants. Plant Cell 13: 1499-510 
Brabbs TR, He Z, Hogg K, Kamenski A, Li Y, Paszkiewicz KH, Moore KA, O’Toole P, Graham IA \& Jones L (2013) The stochastic silencing phenotype of Arabidopsis morc6 mutants reveals a role in efficient RNA -directed DNA methylation. Plant J. 75: 836-846

Breuer C, Stacey NJ, West CE, Zhao Y, Chory J, Tsukaya H, Azumi Y, Maxwell A, Roberts K \& SugimotoShirasu K (2007) BIN4, a Novel Component of the Plant DNA Topoisomerase VI Complex, Is Required for Endoreduplication in Arabidopsis. Plant Cell 19: 3655-3668

Chen Y, Zou T \& Mccormick S (2016) S -Adenosylmethionine Synthetase 3 Is Important for Pollen Tube Growth. Plant Physiol. 172: 244-253

Cohen AL \& Jia S (2015) Non-coding RNAs and the borders of heterochromatin. Wiley Interdiscip. Rev. RNA 5: 835-847

Dekker J \& Misteli T (2015) Long-Range Chromatin Interactions. Cold Spring Harb. Perspect. Biol. 7: a019356

Dinh TT, Gao L, Liu X, Li D, Li SS, Zhao Y, O'Leary M, Le B, Schmitz RJ, Manavella PA, Li SS, Weigel D, Pontes O, Ecker JR, Chen X, Manavella PA, Li SS, Weigel D, Pontes O, Ecker JR, et al (2014) DNA Topoisomerase $1 \alpha$ Promotes Transcriptional Silencing of Transposable Elements through DNA Methylation and Histone Lysine 9 Dimethylation in Arabidopsis. PLoS Genet. 10: e1004446

Dixon JR, Selvaraj S, Yue F, Kim A, Li Y, Shen Y, Hu M, Liu JS \& Ren B (2012) Topological domains in mammalian genomes identified by analysis of chromatin interactions. Nature 485: 376-380

Earley KW, Haag JR, Pontes O, Opper K, Juehne T, Song K \& Pikaard CS (2006) Gateway-compatible vectors for plant functional genomics and proteomics. Plant J. 45: 616-29

Eichten SR, Ellis NA, Makarevitch I, Yeh C-T, Gent JI, Guo L, McGinnis KM, Zhang X, Schnable PS, Vaughn MW, Dawe RK \& Springer NM (2012) Spreading of Heterochromatin Is Limited to Specific Families of Maize Retrotransposons. PLoS Genet. 8: e1003127

Feng S, Cokus SJ, Schubert V, Zhai J, Pellegrini M \& Jacobsen SE (2014) Genome-wide Hi-C Analyses in Wild-Type and Mutants Reveal High-Resolution Chromatin Interactions in Arabidopsis. Mol. Cell 55: $694-707$

Fransz P \& De Jong H (2011) From nucleosome to chromosome: A dynamic organization of genetic information. Plant J. 66: 4-17

Fransz P, de Jong JH, Lysak M, Castiglione MR \& Schubert I (2002) Interphase chromosomes in Arabidopsis are organized as well defined chromocenters from which euchromatin loops 
emanate. Proc. Natl. Acad. Sci. 99: 14584-14589

Gaspar JM \& Hart RP (2017) DMRfinder: Efficiently identifying differentially methylated regions from MethylC-seq data. BMC Bioinformatics 18: 1-8

Gent JI, Madzima TF, Bader R, Kent MR, Zhang X, Stam M, McGinnis KM \& Dawe RK (2014) Accessible DNA and relative depletion of H3K9me2 at maize loci undergoing RNA-directed DNA methylation. Plant Cell 26: 4903-17

Gilroy KL \& Austin CA (2011) The Impact of the C-Terminal Domain on the Interaction of Human DNA Topoisomerase II $\alpha$ and $\beta$ with DNA. PLoS One 6: e14693

Grob S, Schmid MW \& Grossniklaus U (2014) Hi-C Analysis in Arabidopsis Identifies the KNOT, a Structure with Similarities to the flamenco Locus of Drosophila. Mol. Cell 55: 678-693

Grob S, Schmid MW, Luedtke NW, Wicker T \& Grossniklaus U (2013) Characterization of chromosomal architecture in Arabidopsis by chromosome conformation capture. Genome Biol. 14: R129

Hartung F, Angelis KJ, Meister A, Schubert I, Melzer M \& Puchta H (2002) An archaebacterial topoisomerase homolog not present in other eukaryotes is indispensable for cell proliferation of plants. Curr. Biol. 12: 1787-1791

Hartung F \& Puchta H (2001) Molecular characterization of homologues of both subunits A (SPO11) and B of the archaebacterial topoisomerase 6 in plants. Gene 271: 81-86

Heger P, Marin B, Bartkuhn M, Schierenberg E \& Wiehe T (2012) The chromatin insulator CTCF and the emergence of metazoan diversity. Proc. Natl. Acad. Sci. 109: 17507-17512

Huang W, Loganantharaj R, Schroeder B, Fargo D \& Li L (2013) PAVIS: A tool for Peak Annotation and Visualization. Bioinformatics 29: 3097-3099

Jain M, Tyagi AK \& Khurana JP (2006) Overexpression of putative topoisomerase 6 genes from rice confers stress tolerance in transgenic Arabidopsis plants. FEBS J. 273: 5245-5260

Jain M, Tyagi AK \& Khurana JP (2008) Constitutive expression of a meiotic recombination protein gene homolog, OsTOP6A1, from rice confers abiotic stress tolerance in transgenic Arabidopsis plants. Plant Cell Rep. 27: 767-778

Jamieson K, Wiles ET, McNaught KJ, Sidoli S, Leggett N, Shao Y, Garcia BA \& Selker EU (2016) Loss of HP1 causes depletion of H3K27me3 from facultative heterochromatin and gain of H3K27me2 at constitutive heterochromatin. Genome Res. 26: 97-107 
Jiang H, Moreno-Romero J, Santos-González J, De Jaeger G, Gevaert K, Van De Slijke E \& Köhler C (2017) Ectopic application of the repressive histone modification H3K9me2 establishes postzygotic reproductive isolation in Arabidopsis thaliana. Genes Dev. 31: 1272-1287

Jin Y, Tam OH, Paniagua E \& Hammell M (2015) TEtranscripts: A package for including transposable elements in differential expression analysis of RNA-seq datasets. Bioinformatics 31: 3593-3599

Katoh Y, Ikura T, Hoshikawa Y, Tashiro S, Ito T, Ohta M, Kera Y, Noda T \& Igarashi K (2011) Methionine Adenosyltransferase II Serves as a Transcriptional Corepressor of Maf Oncoprotein. Mol. Cell 41: 554-566

Keller C, Kulasegaran-Shylini R, Shimada Y, Hotz H-R \& Bühler M (2013) Noncoding RNAs prevent spreading of a repressive histone mark. Nat. Struct. Mol. Biol. 20: 994-1000

Kera Y, Katoh Y, Ohta M, Matsumoto M, Takano-Yamamoto T \& Igarashi K (2013) Methionine Adenosyltransferase II-dependent Histone H3K9 Methylation at the COX-2 Gene Locus. J. Biol. Chem. 288: 13592-13601

Kirik V, Schrader A, Uhrig JF \& Hulskamp M (2007) MIDGET unravels functions of the Arabidopsis topoisomerase VI complex in DNA endoreduplication, chromatin condensation, and transcriptional silencing. Plant Cell 19: 3100-3110

Krueger F \& Andrews SR (2011) Bismark: a flexible aligner and methylation caller for Bisulfite-Seq applications. Bioinformatics 27: 1571-1572

Lamesch P, Berardini TZ, Li D, Swarbreck D, Wilks C, Sasidharan R, Muller R, Dreher K, Alexander DL, Garcia-Hernandez M, Karthikeyan AS, Lee CH, Nelson WD, Ploetz L, Singh S, Wensel A \& Huala E (2012) The Arabidopsis Information Resource (TAIR): Improved gene annotation and new tools. Nucleic Acids Res. 40: 1202-1210

Lang Z, Lei M, Wang X, Tang K, Miki D, Zhang H, Mangrauthia SK, Liu W, Nie W, Ma G, Yan J, Duan CG, Hsu CC, Wang C, Tao WA, Gong Z \& Zhu JK (2015) The Methyl-CpG-Binding Protein MBD7 Facilitates Active DNA Demethylation to Limit DNA Hyper-Methylation and Transcriptional Gene Silencing. Mol. Cell 57: 971-983

Langmead B \& Salzberg SL (2012) Fast gapped-read alignment with Bowtie 2. Nat. Methods 9: 357359

Li Q, Gent JI, Zynda G, Song J, Makarevitch I, Hirsch CD, Hirsch CN, Dawe RK, Madzima TF, McGinnis KM, Lisch D, Schmitz RJ, Vaughn MW \& Springer NM (2015) RNA-directed DNA methylation enforces boundaries between heterochromatin and euchromatin in the maize genome. Proc. 
Natl. Acad. Sci. 112: 14728-14733

Liang CS, Hartwig B, Perera P, Mora-garcía S, De Leau E, Thornton H, de Lima Alves F, Rappsilber J, Yang S, James GV, Schneeberger K, Finnegan EJ, Turck F \& Goodrich J (2015) Kicking against the PRCs - A Domesticated Transposase Antagonises Silencing Mediated by Polycomb Group Proteins and Is an Accessory Component of Polycomb Repressive Complex 2. PLoS Genet. 11: e1005660

Lippman Z, Gendrel A-V, Black M, Vaughn MW, Dedhia N, Richard McCombie W, Lavine K, Mittal V, May B, Kasschau KD, Carrington JC, Doerge RW, Colot V \& Martienssen R (2004) Role of transposable elements in heterochromatin and epigenetic control. Nature 430: 471-476

Liu C, Cheng Y-J, Wang J-W \& Weigel D (2017) Prominent topologically associated domains differentiate global chromatin packing in rice from Arabidopsis. Nat. Plants 3: 742-748

Liu C, Wang C, Wang G, Becker C, Zaidem M \& Weigel D (2016) Genome-wide analysis of chromatin packing in Arabidopsis thaliana at single-gene resolution. Genome Res. 26: 1057-1068

Liu C \& Weigel D (2015) Chromatin in 3D: progress and prospects for plants. Genome Biol. 16: 170

Liu X, Gao L, Dinh TT, Shi T, Li D, Wang R, Guo L, Xiao L \& Chen X (2014) DNA Topoisomerase I Affects Polycomb Group Protein-Mediated Epigenetic Regulation and Plant Development by Altering Nucleosome Distribution in Arabidopsis. Plant Cell 26: 2803-2817

Lorkovi ZJ, Naumann U, Matzke AJM \& Matzke M (2012) Involvement of a GHKL ATPase in RNADirected DNA Methylation in Arabidopsis thaliana. Curr. Biol. 22: 933-938

Lu Y, Shan G, Xue J, Chen C \& Zhang C (2016) Defining the multivalent functions of CTCF from chromatin state and three-dimensional chromatin interactions. Nucleic Acids Res. 44: 62006212

Manohar M, Choi HW, Manosalva P, Austin CA, Peters JE \& Klessig DF (2017) Plant and human MORC proteins have DNA modifying activities similar to type II topoisomerases, but require additional factor(s) for full activity. Mol. Plant. Microbe. Interact. 30: 87-100

Mathieu O, Probst A V \& Paszkowski J (2005) Distinct regulation of histone H3 methylation at lysines 27 and 9 by CpG methylation in Arabidopsis. EMBO J. 24: 2783-91

McCarthy DJ, Chen Y \& Smyth GK (2012) Differential expression analysis of multifactor RNA-Seq experiments with respect to biological variation. Nucleic Acids Res. 40: 4288-4297

Meczes EL, Gilroy KL, West KL \& Austin C a (2008) The Impact of the Human DNA Topoisomerase II C- 
Terminal Domain on Activity. PLoS One 3: e1754

Meteignier LV, Zhou J, Cohen M, Bhattacharjee S, Brosseau C, Caamal Chan MG, Robatzek S \& Moffett P (2016) NB-LRR signaling induces translational repression of viral transcripts and the formation of RNA processing bodies through mechanisms differing from those activated by UV stress and RNAi. J. Exp. Bot. 67: 2353-2366

Mittal A, Balasubramanian R, Cao J, Singh P, Subramanian S, Hicks G, Nothnagel EA, Abidi N, Janda J, Galbraith DW \& Rock CD (2014) TOPOISOMERASE 6B is involved in chromatin remodelling associated with control of carbon partitioning into secondary metabolites and cell walls, and epidermal morphogenesis in Arabidopsis. J. Exp. Bot. 65: 4217-4239

Moissiard G, Cokus SJ, Cary J, Feng S, Billi AC, Stroud H, Husmann D, Zhan Y, Lajoie BR, McCord RP, Hale CJ, Feng W, Michaels SD, Frand AR, Pellegrini M, Dekker J, Kim JK \& Jacobsen SE (2012) MORC Family ATPases Required for Heterochromatin Condensation and Gene Silencing. Science. 336: 1448-1451

Muller HJ (1930) Types of visible variations induced by X-rays in Drosophila. J. Genet. 22: 299-334 Onoda A, Hosoya O, Sano K, Kiyama K, Kimura H, Kawano S, Furuta R, Miyaji M, Tsutsui K \& Tsutsui KM (2014) Nuclear dynamics of topoisomerase II $\beta$ reflects its catalytic activity that is regulated by binding of RNA to the C-terminal domain. Nucleic Acids Res. 42: 9005-9020

Pohl A \& Beato M (2014) Bwtool: A tool for bigWig files. Bioinformatics 30: 1618-1619

Pommier Y, Sun Y, Huang S-YN \& Nitiss JL (2016) Roles of eukaryotic topoisomerases in transcription, replication and genomic stability. Nat. Rev. Mol. Cell Biol. 17: 703-721

Del Prete S, Arpón J, Sakai K, Andrey P \& Gaudin V (2014) Nuclear Architecture and Chromatin Dynamics in Interphase Nuclei of Arabidopsis thaliana. Cytogenet. Genome Res. 143: 28-50

De Preter K, Barriot R, Speleman F, Vandesompele J \& Moreau Y (2008) Positional gene enrichment analysis of gene sets for high-resolution identification of overrepresented chromosomal regions. Nucleic Acids Res. 36: 1-6

R Development Core Team (2017) R: A Language and Environment for Statistical Computing. $R$ Found. Stat. Comput. Vienna, Austria: https://www.r-project.org/

Ramírez F, Dündar F, Diehl S, Grüning BA \& Manke T (2014) deepTools: a flexible platform for exploring deep-sequencing data. Nucleic Acids Res. 42: W187-91

Robinson JT, Thorvaldsdottir H, Winckler W, Guttman M, Lander ES, Getz G \& Mesirov JP (2011) 
Integrative genomics viewer. Nat. Biotechnol. 29: 24-26

Roudier F, Ahmed I, Bérard C, Sarazin A, Mary-Huard T, Cortijo S, Bouyer D, Caillieux E, DuvernoisBerthet E, Al-Shikhley L, Giraut L, Després B, Drevensek S, Barneche F, Dèrozier S, Brunaud V, Aubourg S, Schnittger A, Bowler C, Martin-Magniette M-L, et al (2011) Integrative epigenomic mapping defines four main chromatin states in Arabidopsis. EMBO J. 30: 1928-1938

Saze H, Shiraishi A, Miura A \& Kakutani T (2008) Control of genic DNA methylation by a jmjC domaincontaining protein in Arabidopsis thaliana. Science 319: 462-5

Schneider K, Mathur J, Boudonck K, Wells B, Dolan L \& Roberts K (1998) The ROOT HAIRLESS 1 gene encodes a nuclear protein required for root hair initiation in Arabidopsis. Genes Dev. 12: 20132021

Schneider K, Wells B, Dolan L \& Roberts K (1997) Structural and genetic analysis of epidermal cell differentiation in Arabidopsis primary roots. Development 124: 1789-1798

Schoft VK, Chumak N, Mosiolek M, Slusarz L, Komnenovic V, Brownfield L, Twell D, Kakutani T \& Tamaru H (2009) Induction of RNA-directed DNA methylation upon decondensation of constitutive heterochromatin. EMBO Rep. 10: 1015-1021

Sequeira-Mendes J, Araguez I, Peiro R, Mendez-Giraldez R, Zhang X, Jacobsen SE, Bastolla U \& Gutierrez C (2014) The Functional Topography of the Arabidopsis Genome Is Organized in a Reduced Number of Linear Motifs of Chromatin States. Plant Cell 26: 2351-2366

Sequeira-Mendes J \& Gutierrez C (2016) Genome architecture: from linear organisation of chromatin to the 3D assembly in the nucleus. Chromosoma 125: 455-469

Shen L, Shao N, Liu X, Maze I, Feng J \& Nestler EJ (2013) diffReps : Detecting Differential Chromatin Modification Sites from ChIP-seq Data with Biological Replicates. PLoS One 8: 1-13

Simkova K, Moreau F, Pawlak P, Vriet C, Baruah A, Alexandre C, Hennig L, Apel K \& Laloi C (2012) Integration of stress-related and reactive oxygen species-mediated signals by Topoisomerase $\mathrm{VI}$ in Arabidopsis thaliana. Proc. Natl. Acad. Sci. 109: 16360-16365

Simon L, Voisin M, Tatout C \& Probst AV (2015) Structure and function of centromeric and pericentromeric heterochromatin in Arabidopsis thaliana. Front. Plant Sci. 6: 1-8

Singer SD, Hily JM \& Cox KD (2011) Analysis of the enhancer-blocking function of the TBS element from Petunia hybrida in transgenic Arabidopsis thaliana and Nicotiana tabacum. Plant Cell Rep. 30: 2013-2025 
Soppe WJJ, Jasencakova Z, Houben A, Kakutani T, Meister A, Huang MS, Jacobsen SE, Schubert I \& Fransz PF (2002) DNA methylation controls histone H3 lysine 9 methylation and heterochromatin assembly in Arabidopsis. EMBO J. 21: 6549-6559

Stroud H, Do T, Du J, Zhong X, Feng S, Johnson L, Patel DJ \& Jacobsen SE (2014) Non-CG methylation patterns shape the epigenetic landscape in Arabidopsis. Nat. Struct. Mol. Biol. 21: 64-72

Stunnenberg R, Kulasegaran-Shylini R, Keller C, Kirschmann MA, Gelman L \& Bu hler M (2015) H3K9 methylation extends across natural boundaries of heterochromatin in the absence of an HP1 protein. EMBO J. 34: 2789-2803

Sugimoto-Shirasu K, Roberts GR, Stacey NJ, McCann MC, Maxwell A \& Roberts K (2005) RHL1 is an essential component of the plant DNA topoisomerase VI complex and is required for ploidydependent cell growth. Proc. Natl. Acad. Sci. 102: 18736-18741

Sugimoto-Shirasu K, Stacey NJ, Corsar J, Roberts K \& McCann MC (2002) DNA Topoisomerase VI Is Essential for Endoreduplication in Arabidopsis. Curr. Biol. 12: 1782-1786

Thorvaldsdóttir H, Robinson JT \& Mesirov JP (2013) Integrative Genomics Viewer (IGV): Highperformance genomics data visualization and exploration. Brief. Bioinform. 14: 178-192

Underwood CJ, Henderson IR \& Martienssen RA (2017) Genetic and epigenetic variation of transposable elements in Arabidopsis. Curr. Opin. Plant Biol. 36: 135-141

Uusküla-Reimand L, Hou H, Samavarchi-Tehrani P, Rudan MV, Liang M, Medina-Rivera A, Mohammed H, Schmidt D, Schwalie P, Young EJ, Reimand J, Hadjur S, Gingras A \& Wilson MD (2016) Topoisomerase II beta interacts with cohesin and CTCF at topological domain borders. Genome Biol. 17: 182

Veluchamy A, Jégu T, Ariel F, Latrasse D, Mariappan KG, Kim S-K, Crespi M, Hirt H, Bergounioux C, Raynaud C \& Benhamed M (2016) LHP1 Regulates H3K27me3 Spreading and Shapes the ThreeDimensional Conformation of the Arabidopsis Genome. PLoS One 11: e0158936

Vergara Z \& Gutierrez C (2017) Emerging roles of chromatin in the maintenance of genome organization and function in plants. Genome Biol. 18: 96

Wang C, Liu C, Roqueiro D, Grimm D, Schwab R, Becker C, Lanz C \& Weigel D (2015a) Genome-wide analysis of local chromatin packing in Arabidopsis thaliana. Genome Res. 25: 246-256

Wang J, Lawry ST, Cohen AL \& Jia S (2014) Chromosome boundary elements and regulation of heterochromatin spreading. Cell. Mol. Life Sci. 71: 4841-4852 
Wang J, Vicente-García C, Seruggia D, Moltó E, Fernandez-Miñán A, Neto A, Lee E, Gómez-Skarmeta JL, Montoliu L, Lunyak V V. \& Jordan IK (2015b) MIR retrotransposon sequences provide insulators to the human genome. Proc. Natl. Acad. Sci. 112: E4428-E4437

Wendte JM, Zhang Y, Ji L, Shi X, Hazarika RR, Shahryary Y, Johannes F \& Schmitz RJ (2019) Epimutations are associated with CHROMOMETHYLASE 3-induced de novo DNA methylation. Elife 8:

Witcher M \& Emerson BM (2009) Epigenetic Silencing of the p16INK4a Tumor Suppressor Is Associated with Loss of CTCF Binding and a Chromatin Boundary. Mol. Cell 34: 271-284

Ye T, Krebs AR, Choukrallah M, Keime C, Plewniak F, Davidson I \& Tora L (2011) seqMINER : an integrated ChIP-seq data interpretation platform. 39: 1-10

Yin Y, Cheong H, Friedrichsen D, Zhao Y, Hu J, Mora-Garcia S \& Chory J (2002) A crucial role for the putative Arabidopsis topoisomerase VI in plant growth and development. Proc. Natl. Acad. Sci. 99: 10191-10196

Yoo S, Cho Y \& Sheen J (2007) Arabidopsis mesophyll protoplasts : a versatile cell system for transient gene expression analysis. Nat. Protoc. 2: 1565-1573

Yoon HJ, Hossain S, Held M, Hou H, Kehl M, Tromas A, Sato S, Tabata S, Andersen SU, Stougaard J, Ross L \& Szczyglowski K (2014) Lotus japonicus SUNERGOS1 encodes a predicted subunit A of a DNA topoisomerase VI that is required for nodule differentiation and accommodation of rhizobial infection. Plant J. 78: 811-821

Yusufzai TM, Tagami H, Nakatani Y \& Felsenfeld G (2004) CTCF Tethers an Insulator to Subnuclear Sites, Suggesting Shared Insulator Mechanisms across Species. Mol. Cell 13: 291-298

Zemach A, Kim MY, Hsieh PH, Coleman-Derr D, Eshed-Williams L, Thao K, Harmer SL \& Zilberman D (2013) The arabidopsis nucleosome remodeler DDM1 allows DNA methyltransferases to access H1-containing heterochromatin. Cell 153: 193-205

Zhang P (2005) MetaCyc and AraCyc. Metabolic Pathway Databases for Plant Research. Plant Physiol. 138: $27-37$

Zhang X, Henriques R, Lin S, Niu Q \& Chua N (2006) Agrobacterium -mediated transformation of Arabidopsis thaliana using the floral dip method. Nat. Protoc. 1: 1-6 